Draft VERsion February 26, 2021

Typeset using LATEX twocolumn style in AASTeX63

\title{
The Far Ultraviolet M-dwarf Evolution Survey. I. The Rotational Evolution of High-Energy Emissions*
}

\author{
J. Sebastian Pineda, ${ }^{1,2, \dagger}$ Allison Youngblood, ${ }^{1,3}$ and Kevin France ${ }^{1}$ \\ ${ }^{1}$ University of Colorado Boulder, Laboratoy for Atmospheric and Space Physics, 3665 Discovery Drive, Boulder CO, 80303, USA \\ ${ }^{2}$ Visiting astronomer, Cerro Tololo Inter-American Observatory, National Optical Astronomy Observatory, which is operated by the Association of Universities \\ for Research in Astronomy (AURA) under a cooperative agreement with the National Science Foundation. \\ ${ }^{3}$ NASA Goddard Space Flight Center, 8800 Greenbelt Rd, Greenbelt, MD 20771, USA
}

\begin{abstract}
M-dwarf stars are prime targets for exoplanet searches because of their close proximity and favorable properties for both planet detection and characterization. However, the potential habitability and atmospheric characterization of these exoplanetary systems depends critically on the history of high-energy stellar radiation from $\mathrm{X}$-rays to NUV, which drive atmospheric mass loss and photochemistry in the planetary atmospheres. With the Far Ultraviolet M-dwarf Evolution Survey (FUMES) we have assessed the evolution of the FUV radiation, specifically 8 prominent emission lines, including $\operatorname{Ly} \alpha$, of M-dwarf stars with stellar rotation period and age. We demonstrate tight power-law correlations between the spectroscopic FUV features, and measure the intrinsic scatter of the quiescent FUV emissions. The luminosity evolution with rotation of these spectroscopic features is well described by a broken power-law, saturated for fast rotators, and decaying with increasing Rossby number, with a typical power-law slope of -2 , although likely shallower for Ly $\alpha$. Our regression fits enable FUV emission line luminosity estimates relative to bolometric from known rotation periods to within $\sim 0.3$ dex, across 8 distinct UV emission lines, with possible trends in the fit parameters as a function of source layer in the stellar atmosphere. Our detailed analysis of the UV luminosity evolution with age further shows that habitable zone planets orbiting lower-mass stars experience much greater high-energy radiative exposure relative the same planets orbiting more massive hosts. Around early-to-mid M-dwarfs these exoplanets, at field ages, accumulate up to 10-20× more EUV energy relative to modern Earth. Moreover, the bulk of this UV exposure likely takes place within the first Gyr of the stellar lifetime.
\end{abstract}

\section{INTRODUCTION}

The presence of self-sustained magnetic fields in low-mass stars has important consequences for their upper atmospheric structure and their high-energy radiative environments. Due to non-thermal magnetic heating processes (see within Linsky 1980; Hall 2008), thought to be either wave dissipation (e.g., Narain \& Ulmschneider 1996) or Joule heating from magnetic reconnection (e.g., Klimchuk 2006), these low-mass stars exhibit significant temperature inversions in their outer atmospheres. These portions of the stellar atmosphere, the chromosphere, transition region and corona, are largely responsible for the entire high-energy spectrum from the near ultraviolet (NUV) to the X-rays in low-mass stars (see Linsky 2017, for recent review). While much effort has been devoted to studying stellar chromospheres/coronae, the nature of these structures, the underlying processes that gen-

\footnotetext{
* This research is based on observations made with the NASA/ESA Hubble Space Telescope obtained from the Space Telescope Science Institute, which is operated by the Association of Universities for Research in Astronomy, Inc., under NASA contract NAS 5-26555.

$\dagger$ sebastian.pineda@lasp.colorado.edu
}

erate them, and their evolution remain poorly understood, especially for M-dwarf stars.

Addressing these questions has attained renewed urgency given the prevalence of terrestrial exoplanets orbiting $\mathrm{M}$ dwarfs, with at least $\sim 20 \%$ of these stars hosting an Earthsized planet within their habitable zones (HZ; e.g., Dressing \& Charbonneau 2015; Vanderburg et al. 2020). Moreover, the best systems for detailed atmospheric characterization with the James Webb Space Telescope will be around nearby low-mass M-dwarfs (e.g., Morley et al. 2017). Understanding the high-energy emissions of these systems is crucial because of the role they play in planetary atmospheric mass-loss and photochemistry (e.g., Scalo et al. 2007; Owen \& Jackson 2012; Tian \& Ida 2015; Luger \& Barnes 2015). For example, for a Neptune/Earth-sized planet in the HZ of an M-dwarf, strong radiation shortward of $\lesssim 911 \AA$ (X-rays + extreme ultraviolet, XUV) can dictate the ultimate water content of the planet through atmospheric evaporation (Owen \& Jackson 2012). Furthermore, the balance of NUV (1700$3200 \AA$ ) to FUV (912-1700 ̊) emissions can determine equilibrium levels of abiotically produced $\mathrm{O}_{2}$, complicating the search for biosignatures (see within Meadows et al. 2018). 
The completion of the MUSCLES Treasury Survey provided the first comprehensive constraints of M-dwarf X-ray and UV luminosities from panchromatic observations of exoplanet hosting M-dwarfs (France et al. 2016; Youngblood et al. 2016; Loyd et al. 2016; Youngblood et al. 2017). Their work further showed that the entire XUV and FUV broadband fluxes could be estimated based on a couple of FUV or NUV spectroscopic features (France et al. 2016; Youngblood et al. 2017). These measurements have since become important inputs into models of planetary atmospheres (e.g., Gao et al. 2015; Ranjan et al. 2017). However, interpreting future atmospheric observations, potential biosignature detections and the ability of such atmospheres to develop life, also depends on the evolution of the planetary atmosphere and hence the evolution of the incident radiation field. The MUSCLES survey focused on older field objects with confirmed planet detections, however, at early ages these M-dwarf hosts likely exhibited much stronger high-energy emissions (e.g., Shkolnik \& Barman 2014), capable of desiccating terrestrial worlds early in their lifetimes (Tian \& Ida 2015).

Understanding this evolution has been challenging because of the difficulty in determining stellar ages in the M-dwarf regime (Guinan et al. 2016). Although the ages of some objects can be determined through membership in clusters or young moving groups (see within Zuckerman \& Song 2004), the vast majority of M-dwarfs lack precise age determinations. Instead, rotation can be used as a proxy for stellar age, as in gyrochronology (e.g., Skumanich 1972; Barnes 2003; Meibom et al. 2015; van Saders et al. 2016), although understanding the angular momentum evolution of M-dwarfs remains a topic of continued work (Barnes 2010; Reiners \& Mohanty 2012; Garraffo et al. 2015; Guinan et al. 2016; Garraffo et al. 2018). Nevertheless, there is a fundamental physical interplay between stellar age, rotation and magnetic activity in low-mass stars (e.g., Skumanich 1972; Noyes et al. 1984; Vidotto et al. 2014), a consequence of the feedback between magnetic field generation in the internal dynamo and angular-momentum loss over time through coronally driven stellar winds.

Indeed, rotation-activity correlations have been used extensively as probes of these magnetic processes and their evolution in M-dwarfs, confirming the strong rotational dependence of the magnetic emissions, even across the fully convective boundary toward late M-dwarfs, and a saturation of the activity at fast rotation rates and young ages (e.g., Pizzolato et al. 2003; Stelzer et al. 2013; Wright \& Drake 2016; Newton et al. 2017; Houdebine et al. 2017; AstudilloDefru et al. 2017; Shulyak et al. 2017; Wright et al. 2018). These studies have focused predominantly on X-rays or optical emission lines like $\mathrm{H} \alpha$ to trace the magnetic activity. The quiescent UV spectra of active M-dwarfs had been largely unexplored except for a few well known flare stars (Rutten et al. 1989; Hawley \& Pettersen 1991; Ayres et al. 2003; Hawley et al. 2003, 2007), limiting our ability to probe the rotational evolution of these features. Understanding these UV emissions is not only important for the incident radiation field impacting planetary atmospheres, but the various emission lines spanning a range of formation temperatures in the FUV spectra serve as unique probes throughout the different layers of the transition region $\left(T_{\mathrm{f}} \sim 10^{4.0-5.2}\right)$, where the temperature in the outer atmosphere is rising rapidly from the chromosphere to the corona (see within Linsky 2017).

These developments motivate the Far Ultraviolet M-dwarf Evolution Survey (FUMES) with the Hubble Space Telescope (HST) to examine the rotational evolution of the FUV spectral features in early-to-mid M-dwarfs, provide important benchmarks for their high energy emission over time, and provide constraints to the chromospheric/coronal structure of active low-mass stars. In this paper, the first of several, we focus on the quiescent emissions of our FUMES sample as a function of rotation/age. In Section 2, we introduce the FUMES sample and assess their stellar properties. In Section 3, we discuss our HST observations and spectral measurements. In Section 4, we examine the rotation-activity correlations of UV emission in low-mass stars incorporating literature data. In Section 5, we discuss the implications of our measurements for the temporal evolution of high-energy emissions around low-mass stars. Lastly, in Section 6 we provide our conclusions and summarize our findings in Section 7 .

\section{SAMPLE AND STELLAR PROPERTIES}

In contrast to previous samples of M-dwarf stars selected for UV observations, either exoplanet hosts (e.g., MUSCLES, France et al. 2013) or known flare stars (e.g., Hawley et al. 2003, 2007), the FUMES target list of 10 objects was chosen to span a range of rotation periods from $\sim 1-55$ $\mathrm{d}$, to trace the rotational evolution of low-mass stars and fill the gap in rotation parameter space between the active flare stars and the slowly rotating exoplanet hosts. The rotation period measurements were typically determined from photometric monitoring (e.g., Messina et al. 2010; Newton et al. 2016) or long-term variability of optical emission lines (e.g., Suárez Mascareño et al. 2015). We also included many objects with known ages from likely membership in a young moving group to facilitate age comparisons and provide multiple benchmarks for high energy emissions at early ages. We further focused on early-to-mid M-dwarf systems because thanks to the success of previous studies like MUSCLES (France et al. 2016), there already exists multiple benchmarks in UV emission for slowly rotating ( $270 \mathrm{~d}$ ) objects in this spectral type range, allowing us to focus on providing the comparison with more active targets. 
Table 1. IR Data Observing Log

\begin{tabular}{|c|c|c|c|c|c|c|c|c|}
\hline Name & UT Date & Instrument & Airmass & A0 Calibrator & Seeing & Exp. Time (s) & $\mathrm{SNR}^{a}$ & Weather \\
\hline GJ 4334 & $2017-11-22$ & TSPEC & 1.08 & HD 240290 & $2 "$ & 20 & 160 & Windy, Clear \\
\hline GJ 49 & $2017-11-22$ & TSPEC & 1.15 & HD 5031 & $2 "$ & 8 & 380 & Windy, Clear \\
\hline HIP 112312 & 2017-11-05 & ARCoIRIS & 1.17 & HD 213044 & $1.8^{\prime \prime}$ & 8 & 280 & Clear \\
\hline LP 247-13 & $2017-11-22$ & TSPEC & 1.02 & HD 21038 & $2 "$ & 20 & 275 & Windy, Clear \\
\hline HIP 17695 & 2017-11-05 & ARCoIRIS & 1.24 & HD 24003 & $1.8 "$ & 8 & 360 & Partly Cloudy \\
\hline HIP 23309 & 2017-11-06 & ARCoIRIS & 1.38 & HD 32507 & $1.3 "$ & 10 & 500 & Clear \\
\hline CD-35 2722 & 2017-11-06 & ARCoIRIS & 1.38 & HD 42681 & $1.3 "$ & 8 & 390 & Partly Cloudy \\
\hline GJ 410 & 2019-04-30 & TSPEC & 1.04 & HD101060 & $1.2 "$ & $20-25$ & 170 & Cloudy \\
\hline LP 55-41 & $2017-11-22$ & TSPEC & 1.28 & HD 32781 & $2 "$ & 25 & 120 & Windy, Clear \\
\hline G 249-11 & $2017-11-22$ & TSPEC & 1.3 & HD 32781 & $2 "$ & 25 & 180 & Windy, Clear \\
\hline
\end{tabular}

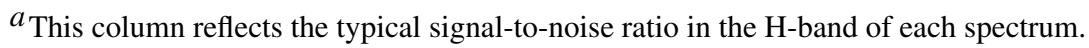

Before delving into the analysis of the UV emissions (Section 3), it is important to estimate the physical properties of our targets. To these ends, we obtained infrared spectra of all 10 stars, which enabled us to consistently determine spectral types, measure metallicity indicators and compare estimates for effective temperature and bolometric luminosity (e.g., Newton et al. 2015; Terrien et al. 2015). These data are discussed in Section 2.1. The IR data were most valuable for estimating spectral types and ruling out the possible influence of cool unknown companions in the photometry. Ultimately, we rely on the results of Pineda et al. (submitted) for the physical properties of the stars used in this work. Those methods are summarized below in Section 2.3.

\subsection{IR Data}

To measure the NIR spectra of our sample targets we used the TripleSpec (TSPEC) instrument (Wilson et al. 2004) on the ARC $3.5 \mathrm{~m}$ telescope at the Apache Point Observatory and the similarly designed ARCoIRIS instrument ${ }^{1}$ on the Blanco $4 \mathrm{~m}$ telescope at NOAO's Cerro Tololo InterAmerican Observatory. A summary of these observations can be found in Table 1. Both instruments, with a 1.1" slit, provide $R \sim 3500$ spectra continuously across NIR wavelengths in 5-6 echelle orders, with TSPEC spanning 0.95-2.46 $\mu \mathrm{m}$ and the updated ARCoIRIS design covering 0.80-2.47 $\mu \mathrm{m}$.

For all of our data we took the same observing approach, using an ABBA slit nod sequence with short exposures $(<30$ s), mitigating sky emission line variability, to provide clean sky subtraction from subsequent frames, and remaining on target over several tens of minutes to obtain a high signalto-noise observation for each target (see Table 1). We also observed a nearby A0 star close in time and at a similar air-

\footnotetext{
${ }^{1}$ Instrument info can be found here: ARCoIRIS
}

mass to provide a reference for flux and telluric calibration (Vacca et al. 2003).

We reduced the data using modified versions of Spextool (Cushing et al. 2004), one for TSPEC and a separate one for ARCoIRIS. ${ }^{2}$ We summarize the data reduction procedure as follows. We first created the master flatfield for each observing night by median combining several dome lamp exposures, and subtracting off the median thermal contribution from dome exposures taken with the lamps off. The thermal contribution is most significant in the $K$-band. The wavelength calibration for each target was then determined from the median sky spectrum, created from each target's science observations. $^{3}$ Initial sky subtraction was performed from differencing $\mathrm{AB}$ nod pairs, from which we determined the object trace in each order. We extracted the spectrum in windows centered along the trace, applying the normalized flatfield and wavelength calibration. When variable cloud cover was evident, we also applied additional sky subtraction, removing a linear fit to the residual background. The spectra from individual frames were averaged together to increase the signal-to-noise ratio and then we used the similarly extracted A0 calibrator spectra to correct for telluric absorption and provide a flux calibration using xtell corr (Vacca et al. 2003). We then merged the different echelle orders, averaging the spectra in overlapping wavelength regions to create the final spectrum of each target. The IR spectra of the FUMES sample is shown in Figure 1.

\subsection{Spectral Types}

\footnotetext{
${ }^{2}$ For TSPEC see TriplespecTool and for the ARCoIRIS version, developed by Dr. Allers, see TS4 Reduction.

${ }^{3}$ Given an $\mathrm{AB}$ nod pair the sky contribution can be estimated as $S=0.5[(A+$ $B)-|(A-B)|]$. For short exposures, one needs to accumulate several frames to produce sufficient signal in sky emission.
} 


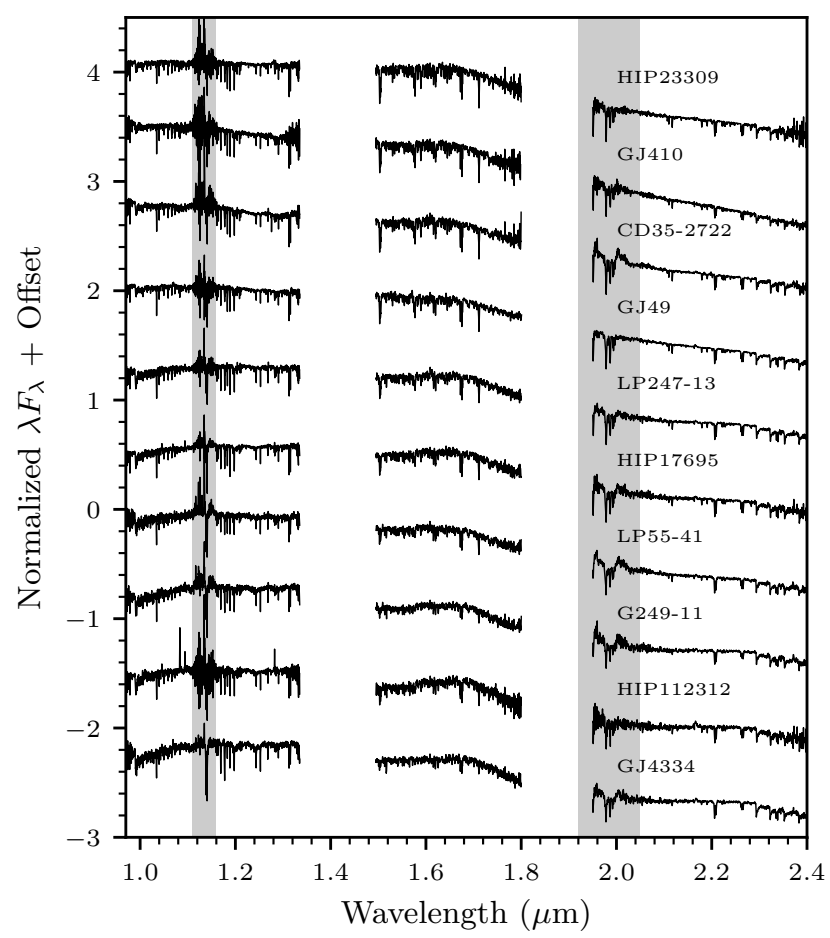

Figure 1. The NIR spectra, shown here, allow us to provide spectral types for our sample in the NIR and assess physical properties using spectroscopic calibrations (see Section 2). The spectra have been normalized in the $H$-band at the median flux between 1.55 and $1.75 \mu \mathrm{m}$. Regions of heavy atmospheric absorption have been removed, with gray shaded areas denoting wavelengths still influenced by some telluric water bands.

We determine the NIR spectral type classifications for the FUMES sample using the composite spectral standards compiled from multiple stars by Newton et al. (2014). ${ }^{4}$ These NIR spectra, taken with the NASA Infrared Telescope Facility (IRTF)/SpeX, are classified on the KHM system originally defined by Kirkpatrick et al. (1991, 1995, 1999) at red optical wavelengths. We first computed the $\mathrm{H}_{2} \mathrm{O}-\mathrm{K} 2$ index defined by Rojas-Ayala et al. (2012) as a $K$-band feature sensitive to spectral type, and used the calibration from Newton et al. (2014) (their Equation 2) to convert this measurement to an initial type estimate. ${ }^{5}$ We then used this classification to select a range of nearby spectral standards, spanning one type earlier and later, to compare against our observations to classify the spectra by eye using features across the entire infrared spectrum. This holistic approach helped mitigate potential feature mismatches introduced by metallicity dif-

\footnotetext{
${ }^{4}$ https://www.dartmouth.edu/ ernewton/nirsurvey.html

${ }^{5}$ We prefer the conversion from $\mathrm{H}_{2} \mathrm{O}-\mathrm{K} 2$ index to NIR spectral type from Newton et al. (2014) rather than Rojas-Ayala et al. (2012) because the former is based on classifications using the entire NIR spectra.
}

ferences between the targets and the standards by not relying on any single features in the spectra. We determined a single best type for each of the $Y J H K$ bands and took the median as our best classification.

Our NIR classifications for the FUMES sample are shown in Table 2, and we estimate spectral type uncertainties of half a subtype. We also include the literature optical spectral types for comparison, typically from the Palomar/Michigan State University (PMSU) survey (Reid et al. 1995; Hawley et al. 1996) for the field objects, but from additional sources for the younger stars. As discussed in Rojas-Ayala et al. (2012) and Newton et al. (2014), the literature optical spectral types are often dependent on metallicity across the M1M4 range, and we consider the NIR classifications to be the more consistent metrics. We similarly report the NIR/optical spectral types for the literature sample in Table 2. These classifications will be important in Section 5.

A couple of objects deserve further attention. Since the Newton et al. (2014) standards do not include spectra for types earlier than M1, we also used the M0 and K7 standards within the IRTF spectral library (Rayner et al. 2009) when classifying GJ410 and HIP23309. The M1 star, CD-35 2722, has an L4 companion that is 5 magnitudes fainter across the $J H K$-bands (Wahhaj et al. 2011) at a separation of $\sim 70 \mathrm{AU}$. This companion is unresolved in our spectroscopic observations, but the $\sim 1 \%$ contribution to the integrated NIR fluxes did not meaningfully distort the observed spectra. However, there was evidence for a slightly deeper $0.99 \mu \mathrm{m}$ FeH feature than would be expected for an M1 dwarf, perhaps due to the strength of this feature in L-dwarf spectra (e.g., Kirkpatrick 2005). The young star HIP 112312 has an optical classification as a subgiant from Torres et al. (2006). Without subgiant standards with which to compare in the NIR, we cannot confirm this classification with our observations. For this star we also compared the M-giant IRTF NIR standards (Rayner et al. 2009), finding that the HIP 112312 NIR spectrum largely agrees with the dwarf sequence except for deeper CO lines in the $K$-band, which are very prominent in the M-giant spectra, reflecting the relatively low gravity of this young object. The other young FUMES stars (see Table 2) showed spectra consistent with the dwarf standards.

\subsection{Stellar Properties}

Our aim in this paper is to analyze the relation between FUV emissions and the physical properties of low-mass stars. To these ends, we desired self-consistent properties, mass, radius, bolometric luminosity, and effective temperature for each FUMES target, and any suitable additional targets found in the literature. Consistently determined properties are crucial to mitigate potential systematic effects introduced by relying on an assortment of eclectic literature determinations for these stellar properties. Although our IR spectra allowed 
Table 2. UV Sample ${ }^{a}$

\begin{tabular}{|c|c|c|c|c|c|c|c|}
\hline Name & $\begin{array}{c}\text { SpT } \\
\text { Opt/NIR }\end{array}$ & $\begin{array}{c}L_{\mathrm{bol}} \\
\left(10^{31} \mathrm{erg} \mathrm{s}^{-1}\right)\end{array}$ & $\begin{array}{l}\text { Mass } \\
\left(M_{\odot}\right)\end{array}$ & $\begin{array}{c}\text { Radius } \\
\left(R_{\odot}\right)\end{array}$ & $\begin{array}{l}T_{\text {eff }} \\
(\mathrm{K})\end{array}$ & $\begin{array}{c}\text { Rot. Period } b \\
\text { (d) }\end{array}$ & References $c$ \\
\hline G 249-11 & $\mathrm{M}^{d} / \mathrm{M} 4$ & $2.59 \pm 0.07$ & $0.237 \pm 0.006$ & $0.255 \pm 0.010$ & $3277 \pm_{68}^{71}$ & 52.76 & $14,14,12$ \\
\hline HIP $112312^{e}$ & M4IV/M4.5 & $16.6 \pm 0.5$ & $0.247 \pm_{0.015}^{0.018}$ & $0.688 \pm_{0.016}^{0.015}$ & $3173 \pm_{22}^{25}$ & $2.355 \pm 0.005$ & $19,14,9$ \\
\hline GJ 4334 & M4.5/M5 & $3.68 \pm 0.09$ & $0.294 \pm 0.007$ & $0.307 \pm 0.012$ & $3260 \pm \pm_{66}^{70}$ & 23.54 & $15,14,12$ \\
\hline LP 55-41 & $\mathrm{M} 3.5^{d} / \mathrm{M} 3$ & $8.10 \pm 0.22$ & $0.41 \pm 0.01$ & $0.416 \pm 0.017$ & $3412 \pm_{73}^{75}$ & 53.44 & $14,14,12$ \\
\hline HIP $17695^{f}$ & M3/M4 & $11.5 \pm 0.2$ & $0.435 \pm_{0.014}^{0.011}$ & $0.502 \pm_{0.007}^{0.008}$ & $3393 \pm_{21}^{20}$ & $3.87 \pm 0.01$ & $1,14,9$ \\
\hline LP 247-13 & M2.7/M3.5 & $12.68 \pm 0.35$ & $0.495 \pm 0.013$ & $0.49 \pm 0.02$ & $3511 \pm_{76}^{79}$ & 1.289 & $16,14,5$ \\
\hline GJ 49 & M1.5/M1 & $18.7 \pm 0.4$ & $0.541 \pm 0.015$ & $0.534 \pm 0.023$ & $3713 \pm_{81}^{86}$ & $18.6 \pm 0.3$ & $15,14,4$ \\
\hline GJ 410 & M0/M0.5 & $21.3 \pm 0.5$ & $0.557 \pm 0.015$ & $0.549 \pm 0.024$ & $3786 \pm_{83}^{89}$ & 14.0 & $15,14,4$ \\
\hline CD-35 2722 $f g$ & M1/M1 & $21.0 \pm 0.3$ & $0.572 \pm 0.002$ & $0.561 \pm 0.003$ & $3727 \pm_{6}^{8}$ & $1.717 \pm 0.004$ & $19,14,9$ \\
\hline HIP $23309^{e}$ & M0/M0 & $68.2 \pm_{1.1}^{1.2}$ & $0.785 \pm_{0.010}^{0.009}$ & $0.932 \pm 0.014$ & $3886 \pm_{27}^{28}$ & $8.6 \pm 0.07$ & $19,14,9$ \\
\hline Prox. Cen. * & M5.5/- & $0.5997 \pm_{0.0075}^{0.0076}$ & $0.123 \pm 0.003$ & $0.147 \pm 0.005$ & $2992 \pm_{47}^{49}$ & 88.977 & $6,-, 13$ \\
\hline GJ 1061 & M5.5/- & $0.628 \pm 0.014$ & $0.125 \pm 0.003$ & $0.152 \pm 0.007$ & $2976 \pm_{72}^{69}$ & 133 & $6,-, 2$ \\
\hline GJ 1214 & $\mathrm{M} 4.5 / \mathrm{M} 4$ & $1.343 \pm_{0.034}^{0.036}$ & $0.181 \pm 0.005$ & $0.204 \pm_{0.0084}^{0.0085}$ & $3111 \pm_{66}^{69}$ & $125 \pm 5$ & $15,11,8$ \\
\hline GJ 1132 & M3.5/- & $1.67 \pm 0.05$ & $0.194 \pm 0.005$ & $0.215 \pm 0.009$ & $3196 \pm \pm_{69}^{72}$ & 129.15 & $6,-, 13$ \\
\hline Gl 213 & M4/M4 & $2.41 \pm 0.04$ & $0.218 \pm 0.005$ & $0.238 \pm 0.009$ & $3334 \pm_{63}^{67}$ & 170 & $15,11,2$ \\
\hline GJ 628 * & M3.5/M3 & $4.21 \pm 0.04$ & $0.304 \pm 0.007$ & $0.319 \pm 0.007$ & $3307 \pm \pm_{36}^{38}$ & $119.3 \pm 0.5$ & $15,11,18$ \\
\hline Gl $581 *$ & M3/M2 & $4.56 \pm 0.04$ & $0.307 \pm 0.007$ & $0.310 \pm 0.008$ & $3424 \pm_{42}^{43}$ & $132.5 \pm 6.3$ & $15,11,17$ \\
\hline YZ CMi & M4.5/M5 & $4.35 \pm_{0.14}^{0.15}$ & $0.316 \pm 0.008$ & $0.328 \pm 0.013$ & $3293 \pm_{71}^{74}$ & $2.7758 \pm 0.0002$ & $15,11,10$ \\
\hline EV Lac & M3.5/M3 & $4.88 \pm 0.15$ & $0.320 \pm 0.008$ & $0.331 \pm 0.013$ & $3370 \pm_{70}^{75}$ & $4.3715 \pm 0.0002$ & $15,11,10$ \\
\hline GJ 667C & M1.5/- & $5.51 \pm 0.13$ & $0.327 \pm 0.008$ & $0.337 \pm 0.014$ & $3443 \pm_{71}^{75}$ & $103.9 \pm 0.7$ & $6,-, 17$ \\
\hline GJ 876 * & M4/M3 & $5.01 \pm 0.04$ & $0.346 \pm 0.007$ & $0.372 \pm 0.004$ & $3201 \pm_{19}^{20}$ & $87.3 \pm 5.7$ & $15,11,17$ \\
\hline G1 821 & M1/- & $6.98 \pm 0.09$ & $0.355 \pm 0.009$ & $0.363 \pm_{0.014}^{0.015}$ & $3512 \pm_{69}^{73}$ & 107 & $15,-, 2$ \\
\hline Gl $436 *$ & $\mathrm{M} 2.5 / \mathrm{M} 3$ & $9.42 \pm 0.11$ & $0.425 \pm 0.009$ & $0.432 \pm 0.011$ & $3477 \pm_{44}^{46}$ & $44.09 \pm 0.08$ & $15,11,3$ \\
\hline AD Leo & M3/M3 & $8.7 \pm 0.1$ & $0.426 \pm 0.010$ & $0.43 \pm 0.02$ & $3425 \pm_{68}^{69}$ & $2.2399 \pm 0.0002$ & $15,11,10$ \\
\hline GJ 832 & M1.5/- & $10.6 \pm 0.3$ & $0.441 \pm 0.011$ & $0.442 \pm 0.018$ & $3539 \pm \pm_{74}^{79}$ & $45.7 \pm 9.3$ & $6,-, 17$ \\
\hline Gl $887 *$ & M0.5/ & $14.08 \pm 0.22$ & $0.48 \pm 0.01$ & $0.474 \pm 0.008$ & $3672 \pm_{34}^{36}$ & 33 & $6,-, 2$ \\
\hline GJ $176 *$ & $\mathrm{M} 2 / \mathrm{M} 2$ & $13.46 \pm 0.12$ & $0.485 \pm 0.012$ & $0.474 \pm 0.015$ & $3632 \pm_{56}^{58}$ & $39.3 \pm 0.1$ & $15,11,17$ \\
\hline $\mathrm{AU} \mathrm{Mic}^{e}$ & M0/- & $37.7 \pm_{0.7}^{0.8}$ & $0.667 \pm_{0.008}^{0.006}$ & $0.798 \pm_{0.013}^{0.014}$ & $3619 \pm_{25}^{23}$ & 4.86 & $6,-, 7$ \\
\hline
\end{tabular}

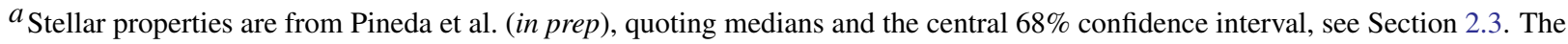
horizontal division separates the new FUMES (top) targets from the literature stars (bottom).

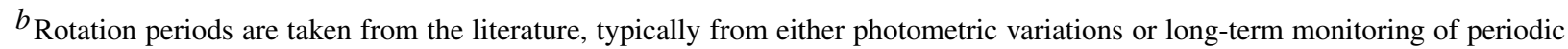
emission lines. Uncertainties are as reported in the literature if available.

${ }^{c}$ References in order denote source for optical spectral type, infrared spectral type and rotation period.

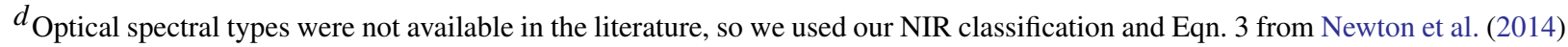
with the metallicity determined from their calibration of the equivalent widths of the Na doublet at $2.2 \mu \mathrm{m}$ (their Equation 10).

${ }^{e}$ HIP 23309, HIP112312, and AU Mic are members of $\beta$ Pic which has a mean age of 24 Myr (Bell et al. 2015).

$f_{\mathrm{CD}-35} 2722$, and HIP17695 are members of AB dor which has a mean age of $\sim 150 \mathrm{Myr}$ (Bell et al. 2015).

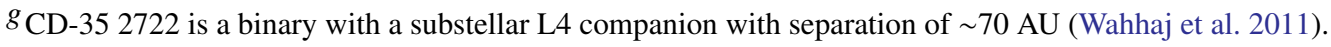

* Star names listed with an asterisk have measured angular diameters from interferometric measurements (von Braun et al. 2011; Boyajian et al. 2012; von Braun et al. 2014; Kane et al. 2017), incorporated in the radius estiamtes, see Pineda et al. (in prep).

References. - (1) Alonso-Floriano et al. (2015), (2) Astudillo-Defru et al. (2017), (3) Bourrier et al. (2018), (4) Donati et al. (2008), (5) Hartman et al. (2011), (6) Hawley et al. (1996), (7) Küker et al. (2019), (8) Mallonn et al. (2018), (9) Messina et al. (2010), (10) Morin et al. (2008), (11) Newton et al. (2014), (12) Newton et al. (2016), (13) Newton et al. (2018), (14) This Work, (15) Reid et al. (1995), (16) Shkolnik et al. (2009), (17) Suárez Mascareño et al. (2015), (18) Suárez Mascareño et al. (2016), (19) Torres et al. (2006). 
us to utilize spectroscopic property calibrations (e.g., Mann et al. 2015; Newton et al. 2015; Terrien et al. 2015), such data were not uniformly available for both the FUMES and literature targets, and we thus rely instead on the largely photometric results from our companion paper summarized below (Pineda et al. in prep). The corresponding stellar properties are shown in Table 2.

\subsubsection{Field Stars}

To determine the properties of low-mass field stars Pineda et al. (in prep) use a Bayesian framework to combine multiple empirical calibrations, largely photometry based, to jointly constrain mass, radius, bolometric luminosity, and derive the stellar effective temperature. Their methods fully incorporate measurement uncertainties, and intrinsic scatter within the utilized calibrations, and produce well defined joint posterior distributions for the full set of physical properties. Pineda et al. (in prep) jointly uses the mass-luminosity relation of Mann et al. (2019), the bolometric correction calibration of Mann et al. (2015), and a new mass-radius relation valid across $0.1-0.7 M_{\odot}$ with $3.1 \%$ uncertainties at fixed mass specifically developed in that work.

The Bayesian framework further allowed them to freely incorporate additional measurements whenever available, such as bolometric fluxes, or angular diameters from interferometry. Many of the individual objects in the sample (see Table 2) had these additional measurements which largely improved the precision of the physical properties of a given sample object. Full details on these methods are available in Pineda et al. (in prep).

As compared to literature estimates of the M-dwarf ensemble analyzed in Pineda et al. (in prep), their methods yielded a stellar sequence with less scatter, consistent property estimates for objects with interferometric angular diameter measurements, and stellar densities consistent with independent data inferred from exoplanetary transits of low eccentricity planets.

\subsubsection{Young Stars}

Of the objects shown in Table 2, five are high probability members of known young moving groups: HIP 112312 , HIP 17695, CD-35 2722, HIP 23309, and AU Mic (Pineda et al. in prep). Because the available empirical calibrations are only applicable to field age stars, these five young objects required a different approach for estimating their stellar properties. For these stars, Table 2 also quotes the stellar model based results from Pineda et al. (in prep). We summarize their methods as follows.

Within a Bayesian framework, Pineda et al. (in prep) couple stellar evolutionary models with spectral energy distribution fitting of model spectra using blue optical to far infrared photometry. Using Monte Carlo sampling, a given mass and age within the evolutionary model defines the corresponding bolometric luminosity and radius, and thus the effective temperature and gravity of a sample point. These properties are then used for interpolation of a model atmosphere grid and generation of synthetic photometry for comparison with the observed data points. The best-fit stellar properties are those that best reproduce the photometry consistently within the evolutionary models. This approach self-consistently produces parameter estimates for all of the properties with well defined posterior distributions for each. For the five young objects, Table 2 reproduces the parameter results using magnetic stellar evolutionary models (Feiden \& Chaboyer 2013, 2014; Feiden 2016). Full details of these methods and analysis of likely systematic effects in the model choices are explained in Pineda et al. (in prep).

Those properties most directly constrained by the SED fitting, namely $L_{\mathrm{bol}}, T_{\mathrm{eff}}$, and $R$ are consistent within errors to literature values (Pineda et al. in prep). The young stars used in this work are all active M-dwarfs, and we discuss further how their modeling choices and thus the inferred mass impact our rotation activity analysis in Section 4.1.

\section{FAR ULTRAVIOLET EMISSIONS}

\subsection{HST Data}

We used the Space Telescope Imaging Spectrograph (STIS) on HST to measure the FUV spectra of our FUMES sample through program HST GO-14640 (PI - Pineda). We show a summary of our observations in Table 3. Typically data were taken using the G140L grating with the FUVMAMA detector, providing a typical resolving power of $\sim 1000$ across $1150-1730 \AA$. For the brighter targets we used the echelle grating E140M instead, with similar wavelength coverage and a resolving power of $\sim 45,000$. Although the lower resolution of the G140L grating does not permit measurement of the typical FUV line-widths, and doublet lines are often blended, our focus in this study is simply the total flux in the strongest FUV lines, which we can measure using G140L for fainter targets than is possible at high resolution using STIS. All observations were taken in photon counting TIME-TAG mode, providing high time resolution to our program. We focus on the quiescent emissions of the FUMES sample here, integrated across all exposures and orbits, after removing flares, with the time resolved analysis to be presented in a follow-up paper.

To extract the spectra from the FUV-MAMA photon counting detector we used the spectralPhoton routines from $\mathrm{R}$. O. P. Loyd previously used in the HST-STIS and HST-COS analyses of M-dwarf UV spectra (e.g., Loyd \& France 2014; Loyd et al. 2018). ${ }^{6}$ To summarize, the reduction procedure

\footnotetext{
${ }^{6}$ The codes are available on GitHub: https:/github.com/parkus/ spectralPhoton.
} 


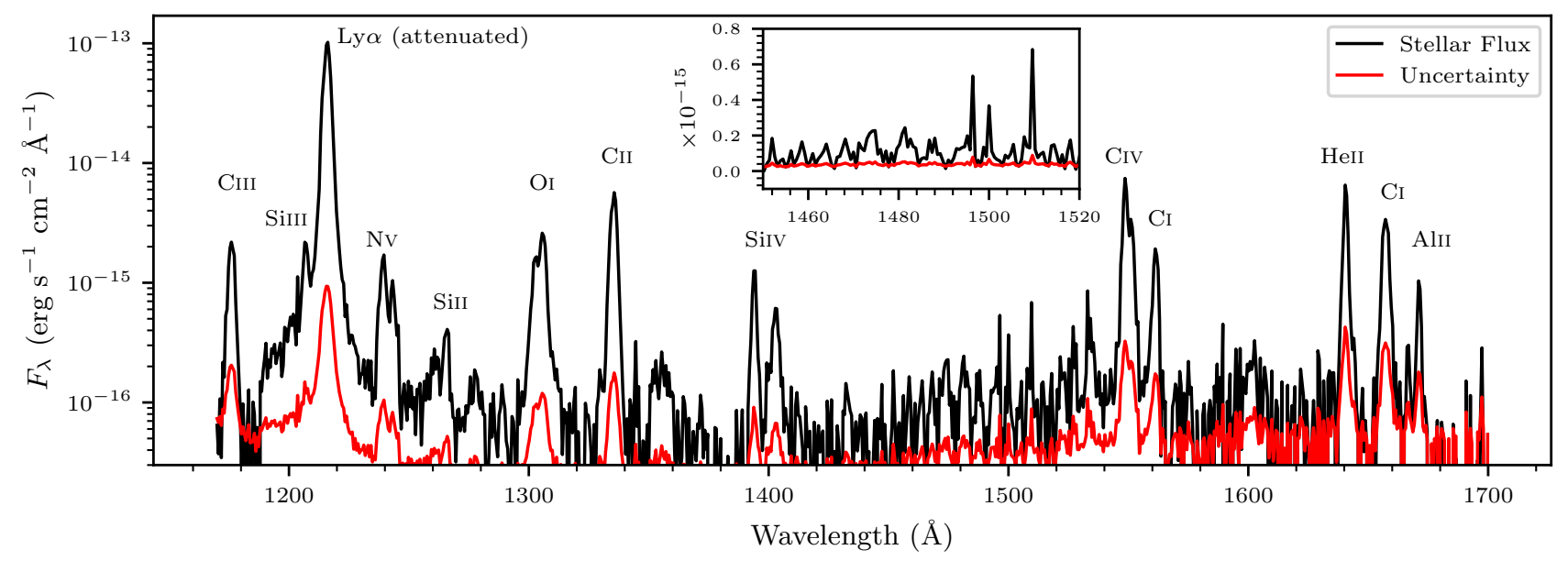

Figure 2. The FUV spectra of M-dwarfs show several discrete emission features spanning lines that probe different temperatures in the transition region from Cir to Nv. This example illustrates a typical spectrum in the FUMES data set (GJ49, $\left.\mathrm{T}_{\exp }=5585 \mathrm{~s}\right)$ spanning $1170-1700 \AA$ using $H S T$-STIS G140L. The inset figure shows the low-level continuum emission from 1450-1520 А.

sums the photons in a narrow ribbon identified as the stellar trace subtracting off a background count rate determined from the signal in offset regions with the same wavelengths. The reduction then uses the flux calibration from the full exposures to convert the photon counts to a calibrated spectrum. The advantage of spectralphoton over the standard output from the STScI pipelines is that it allows for the definition of custom wavelength extraction regions, trace locations, and integrated time intervals. This was important because we found for the faint targets LP 55-41 and G 249-11 that the standard pipeline products did not correctly identify the stellar trace. Additionally, some of the targets flared during our observations, which we removed by manually identifying when the flares took place, and defining custom time intervals during the exposures for extraction of photons corresponding to the quiescent emission spectra.

The majority of our targets were observed in the wider 0.2 " STIS slit mitigating potential slit-loss effects, affecting the flux calibration, with poor acquisition, target centering or guiding. For one target, HIP 23309, observed with the narrower 0.1 " slit for bright object protection considerations, the time series analysis showed a long-term trend in the photon counts over the course of the orbit (this effect was not seen in the data for GJ 410, the other program observation employing the 0.1 " slit). To correct for this we fit a third-order polynomial to the trend (in the $10 \mathrm{~s}$ binned light curve) to divide it out and scaled to the average peak count rate. The quiescent spectrum was subsequently extracted from the appropriately scaled spectrum. For each target we extracted spectra from each exposure (usually 1 per orbit), and then co-added them together rebinning onto a constant linear wavelength grid. For the echelle spectra we also merged the wavelength regions in which the echelle orders overlap, averaging flux points falling within the same $0.05 \AA$ bins, and preserving
Table 3. UV Data Summary

\begin{tabular}{lclcc}
\hline \multicolumn{1}{c}{ Name } & UT Date & Grating & Aperture $^{a}$ & Orbits \\
\hline GJ 4334 & $2017-09-20$ & G140L & $0.2 ”$ & 2 \\
GJ 49 & $2017-09-20$ & G140L & $0.2 ”$ & 2 \\
HIP 112312 & $2017-08-22$ & E140M & $0.2 ”$ & 1 \\
LP 247-13 & $2017-09-13$ & G140L & $0.2 ”$ & 1 \\
HIP 17695 & $2017-12-27$ & E140M & $0.2 ”$ & 1 \\
HIP 23309 & $2017-11-24$ & G140L & $0.1 ”$ & 1 \\
CD-35 2722 & $2017-09-26$ & G140L & $0.2 ”$ & 1 \\
GJ 410 & $2017-12-18$ & G140L & $0.1 ”$ & 1 \\
LP 55-41 & $2017-09-13$ & G140L & $0.2 ”$ & 3 \\
G 249-11 & $2017-09-10$ & G140L & $0.2 ”$ & 3 \\
\hline
\end{tabular}

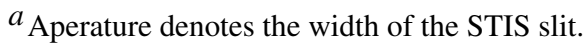

the integrated flux of the spectral bin. We show an example G140L spectrum of one of our targets in Figure 2, and an echelle E140M spectrum in Figure 3.

By using STIS, we were able to observe Ly $\alpha$ in all of our objects, as well as all of the significant FUV emission lines HeII $\lambda 1640.4 \AA$, CII $\lambda \lambda 1334.54,1335.71 \AA$, C CIII $\lambda 1175.7 \AA$, Civ $\lambda \lambda 1548.19,1550.78 \AA$, SiIII $\lambda 1206.5 \AA$, Sirv $\lambda \lambda 1393.76,1402.77 \AA$, and Nv $\lambda \lambda 1238.82,1242.806 \AA$. These lines span mean formation temperatures $\log T(\mathrm{~K})=$ 4.5-5.2, probing the transition region of the stellar coronal atmosphere. ${ }^{7}$ We focused on these lines as both the

\footnotetext{
${ }^{7}$ The mean formation temperatures listed in Table 7 depend on the differential emission measure and may differ (significantly) from the peak formation temperature.
} 

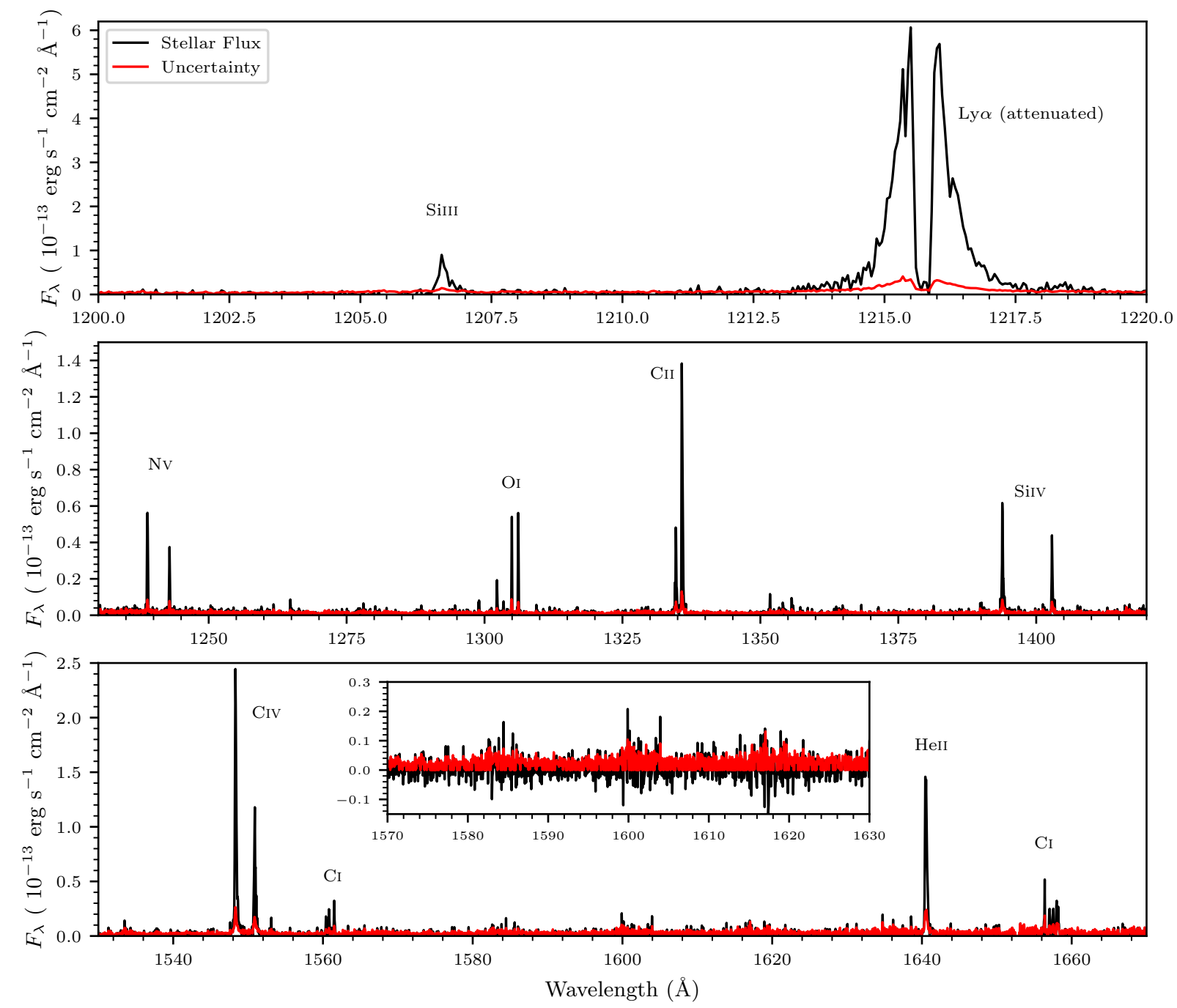

Figure 3. This HST-STIS E140M data for HIP 17695 ( $\mathrm{T}_{\exp }=1974 \mathrm{~s}$ ) shows the discrete FUV emission features of M-dwarfs prominently, with no continuum FUV flux detected. Important emission features are labeled, with the multiplet nature of some of the lines more clearly resolved. The inset figure shows an example continuum emission region from 1570-1630 ̊.

most prominent in the data, being well measured both in the FUMES targets and the literature sample.

\subsection{FUV Line Fitting}

To measure the target emission line fluxes, we used PyMC3 to fit the observed line shapes, typically with either a Voigt or Gaussian profile convolved with the instrument line spread function. ${ }^{8}$ Although we must assume a particular profile shape, these shapes are well motivated physically, and this approach has several advantages to simply summing the flux in the appropriate region for each line. At high resolution, we can measure the line widths, compare the emission core to the line wings, and examine centroid offsets, in-

\footnotetext{
${ }^{8}$ The LSFs have spectral resolutions of 1.7-1.5 pixels at FWHM for STISG140L and 1.4-1.3 pixels at FWHM for E140M. We used the LSFs obtained from the STSci STIS instrument documentation.
}

dicative of the stellar radial velocity. We can also simultaneously fit a continuum level below each line and incorporate the uncertainty in that estimate to our reported emission line fluxes. This effect was especially important in the G140L data, where there appeared to be some continuum level below many of the lines. For example in the region around $\mathrm{Nv}$, some of the low-level extended Ly $\alpha$ wing emission could be seen. In the E140M data, no continua were evident. We quantify continuum levels in Section 3.3. Additionally, the line fitting is robust to potentially badly characterized data points because it allows us to include a fit scatter term to the data to account for underestimated errors.

We summarize the model profiles fit to the data in Table 4, where the number in front of the profile shape indicates that multiple lines were fit simultaneously, and the continuum component is described by a line with an unknown slope and 

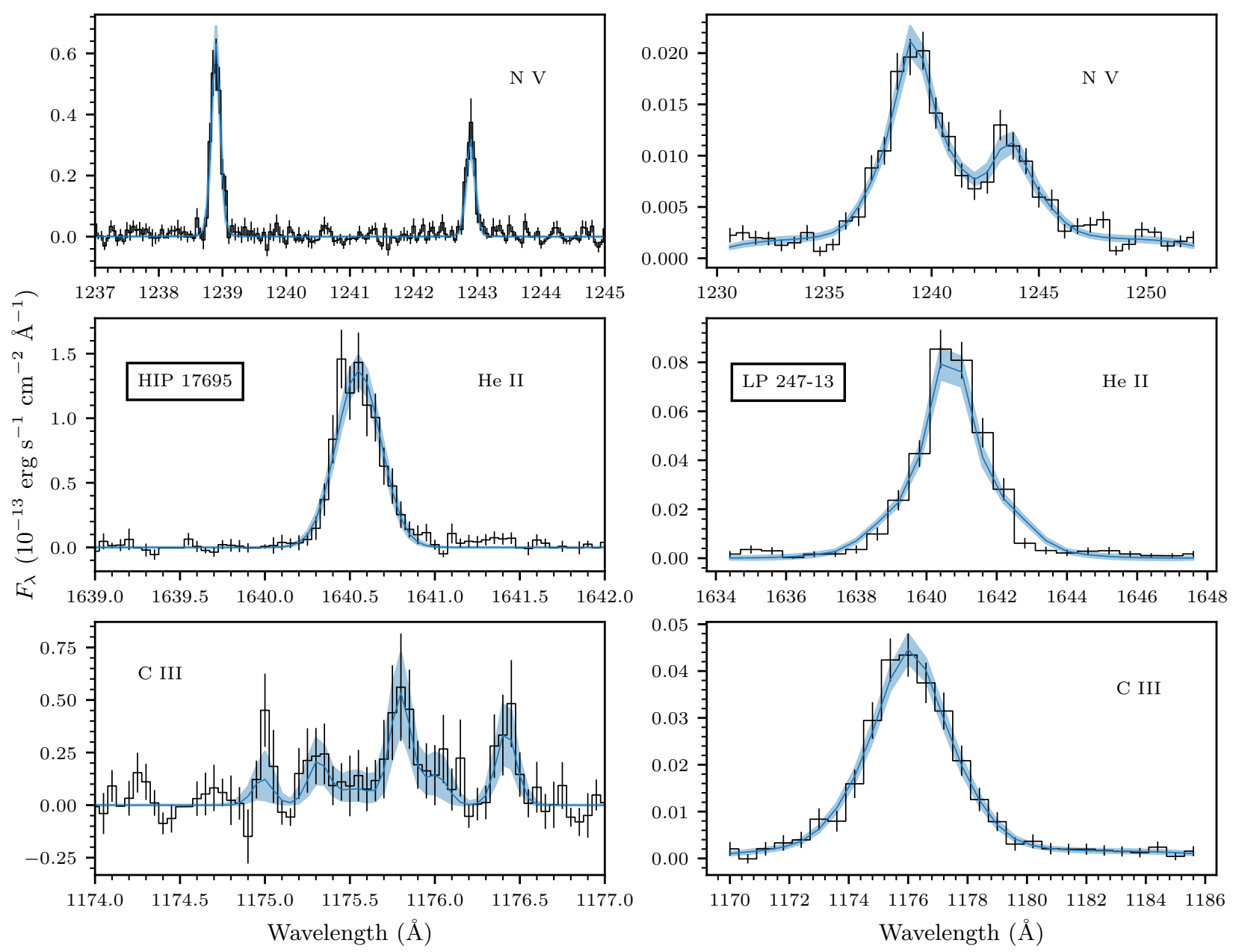

Figure 4. We show example results displaying our typical line fits for Nv (top), HeII (middle), and CIII (bottom), with both E140M high resolution data (left), and G140L low resolution data (right). Data (black) are from HIP 17695 (left) and LP247-13 (right), with the shaded region (blue) denoting the $1 \sigma$ confidence interval for the model about its median. Emission lines that are separable at high resolution can become blended in the lower resolution data.

constant offset. When multiple lines are fit simultaneously, as in the Civ doublet, the widths of the Gaussian and Lorentzian components in the Voigt profile shape are constrained to be identical in each line. This reduces the number of free parameters in the fit and is justified because each line comes from the same species, forming under similar temperature conditions with only a small difference in energy levels between the transitions. As priors in our Bayesian regression analysis, we used uniform distributions for the line flux, center, and when necessary the continuum offset and slope. For the width parameters in the Gaussian and Lorentzian components we used a weakly informative Half-Cauchy distribution, with a characteristic width of $100 \mathrm{~km} \mathrm{~s}^{-1}$. 9 This choice had a negligible affect on the parameter posterior distribu-

\footnotetext{
${ }^{9}$ For the non-negative Half-Cauchy distribution, half of the probability mass density is contained at parameter values less than the characteristic width with the distribution tail decaying in a Lorentzian fashion.
}

tion, but greatly improved MCMC sampling efficiency and convergence relative to a log uniform prior within PyMC3.

We show examples of our fit line profiles in Figure 4, with the results for the total line fluxes shown in Table 5. The Ly $\alpha$ emission is the focus of paper II (Youngblood et al. $a c$ cepted), and we reproduce those results here. For the two targets with E140M data, we fit a single Gaussian line for SiIII, to be consistent with the approach for the G140L data, that required simultaneous fitting with $\operatorname{Ly} \alpha$ (Youngblood et al. accepted). At the higher resolution, the low flux CIII lines are somewhat blended but distinguishable, so we fit six components to the multiplet, but only a single Gaussian feature at low-resolution. The Herr multiplet, by contrast, remains unresolved in all the datasets and we fit a single Gaussian for the combined emission accordingly. In general for the HeII line fits, the best model scatter term was often larger than the fits for other lines of the same star possibly indicating that the shape choice was not ideally suited to the data. For this 
Table 4. Model Line Fitting

\begin{tabular}{|c|c|c|}
\hline Line ID & G140L & E140M \\
\hline $\operatorname{Ly} \alpha^{a}$ & Voigt + ISM & Voigt + ISM \\
\hline HeII & Gaussian + Continuum & Gaussian \\
\hline $\mathrm{C}_{\mathrm{III}} b$ & Gaussian + Continuum & Voigt \\
\hline CIII & Gaussian+ Continuum & 6x Gaussian \\
\hline Civ & $2 \mathrm{x}$ Voigt + Continuum & $2 \mathrm{x}$ Voigt \\
\hline $\mathrm{Nv}$ & $2 \mathrm{x}$ Voigt + Continuum & $2 \mathrm{x}$ Voigt \\
\hline SiIII $a$ & Gaussian $+\operatorname{Ly} \alpha$ & Gaussian \\
\hline Sirv & $2 \mathrm{x}$ Voigt + Continuum & 2x Voigt \\
\hline
\end{tabular}

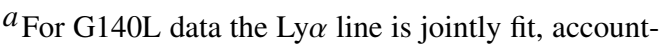
ing for ISM absorption, with SiIII (Youngblood et al. accepted), but for E140M data the SirI line is fit independently.

$b_{\text {For the E140M data we fit only the single redward }}$ line in the CII doublet, $\lambda 1335.71 \AA$, since the $\lambda$ $1334.54 \AA$ line is affected by the ISM; for G140L data, these lines are blended, see Table 5 .

unresolved multiplet, however, our model choice remains the simplest way to capture the line flux.

For the E140M data the Cir doublet is resolved, but at lowresolution the two lines are blended, which impacts the flux measurements since one of the lines is impacted by ISM absorption. We estimated the typical ISM attenuation of the CII $1334 \AA$ A line by assuming typical ISM parameters from Redfield \& Linsky (2004) and a stellar line approximated as a Voigt profile with a width of $30 \mathrm{~km} \mathrm{~s}^{-1}(0.05 \AA)$ for the Gaussian component and $20 \mathrm{~km} \mathrm{~s}^{-1}(0.04 \AA)$ for the Lorentzian component based on our E140M spectra of HIP 17695's unattenuated CII $1335 \AA$ A line. The range of CII ISM parameters identified by Redfield \& Linsky (2004) for stars inside $40 \mathrm{pc}$ is 13.1-15.2 for the log column density, 2.9-6.6 $\mathrm{km} / \mathrm{s}$ for the Doppler $b$ value, and a radial velocity of $-30 \mathrm{~km}$ $\mathrm{s}^{-1}$ to $30 \mathrm{~km} \mathrm{~s}^{-1}$. We created 10,000 realizations of the attenuated $1334 \AA$ Alux where column density, $b$ value, and radial velocity of the absorbers relative to the star's rest frame were drawn from uniform distributions with the ranges described previously. We found that the median attenuation for the $1334 \AA$ line is $14 \%$, with a $68 \%$ confidence interval range of 4-45\%. Assuming the 1334 and $1335 \AA$ CII lines are equally populated (a reasonable assumption for thermal equilibrium), total CII fluxes derived from the low-resolution spectra where the two lines are blended should be corrected upward $~ 7 \%$, with a $68 \%$ confidence interval range of $2-23 \%$.

The STIS data for G249-11 and LP 55-41 were much fainter than the rest of the FUMES targets, and many of the typical emission lines were barely visible. We determined the
$3 \sigma$ upper limits for these lines by simply summing the flux in the region around the expected line center, and computing the associated uncertainty in that flux. For the few lines that were measurable, CII, CIv, and Nv, we accommodated the lower signal-to-noise ratio by only fitting single Gaussian line profiles in each case, one component for the blended CII, and two components each for the $\mathrm{CIv}$ and Nv doublets. These results are included in Table 5 .

\subsection{FUV Continuum Emission}

Although the FUV spectra of low-mass stars is dominated by discrete emission line features, there is a weak underlying FUV continuum that is likely defined by the recombination edges of species like Si (Loyd et al. 2016; Peacock et al. 2019). As seen in Figures 2 and 3, continuum emission between the strong line features was evident in the low-resolution G140L spectra, but unapparent in the highresolution E140M spectra. This is a consequence of the higher sensitivity and lower resolution of the G140L grating, making it easier to detect weak continuum levels. Compared to HST-COS medium-resolution gratings (G130M, G160M), the HST-STIS low-resolution grating (G140L) is slightly more sensitive at detecting faint continuum emission. ${ }^{10}$ For the full FUMES sample we quantify the FUV continuum levels apparent in our spectra following the work of Loyd et al. (2016).

As part of the MUSCLES program they defined an ensemble of narrow bands (0.7-1 $\AA$ in width) interspersed between the prominent FUV emission lines to assess the FUV continuum flux across 1300-1700 ̊. We used these same bands (obtained through private communication, Loyd, R. O. P.) transformed to the radial velocity frame of our targets to integrate the FUV continuum. ${ }^{11}$ We were able to use the bands unaltered for the E140M data sets; however, for the lower resolution G140L data, we visually verified that the bands did not overlap with any of the strong emission lines. This removed 6 narrow bands ( $\sim 6 \AA$ ) that encompassed parts of emission line wings.

The integrated fluxes in these narrow bands are shown in Table 6 , averaged over the $\sim 150 \AA$ cumulative width of the passbands. For 8 of the 10 targets, including the two observed with the E140M grating, we measure a non-zero FUV continuum level, all except for G149-11 and LP 55-41. Although in each narrow band the integrated flux is usually in-

\footnotetext{
${ }^{10}$ Using the STScI exposure time calculator (http://etc.stsci.edu), detecting a low-level flat continuum $\left(F_{\lambda}=10^{-16} \mathrm{erg} \mathrm{s}^{-1} \mathrm{~cm}^{-2} \AA^{-1}\right.$ at $\left.1350 \AA\right)$ at a signal-to-noise threshold of 3 would take twice as long with COS G130M as opposed to STIS G140L (6.1 ks vs. $2.9 \mathrm{ks}$ ) when accounting for the factor of $\sim 15$ difference in resolution.

${ }^{11}$ All the FUMES targets had measured RVs, except G249-11 and LP 55-41, for which we used a $0 \mathrm{~km} \mathrm{~s}^{-1}$ frame. The shift is small in general and has no impact on the result, since these two targets were too faint to detect any continuum emission.
} 
Table 5. FUV Line Fluxes ${ }^{a}$

\begin{tabular}{|c|c|c|c|c|c|c|c|c|}
\hline Name & $\operatorname{Ly} \alpha$ & HeII & $\mathrm{C}_{\mathrm{II}}{ }^{c}$ & CIII & Civ & $\mathrm{Nv}$ & $\mathrm{SiIII}^{d}$ & Sirv \\
\hline G 249-11 & - & $<0.012$ & $<0.012$ & $<0.014$ & $0.013 \pm 0.003$ & $0.0065 \pm 0.0012$ & - & $<0.01$ \\
\hline HIP 112312 & $214 \pm_{6}^{7}$ & $9.14 \pm 0.48$ & $3.97 \pm 0.18$ & $3.01 \pm_{0.47}^{0.53}$ & $11.87 \pm_{0.53}^{0.52}$ & $1.90 \pm_{0.13}^{0.14}$ & $1.67 \pm_{0.28}^{0.29}$ & $2.20 \pm_{0.14}^{0.13}$ \\
\hline GJ 4334 & $7.03 \pm_{1.16}^{3.93}$ & $0.280 \pm_{0.035}^{0.036}$ & $0.68 \pm 0.03$ & $0.15 \pm 0.01$ & $1.62 \pm_{0.06}^{0.07}$ & $0.337 \pm 0.024$ & $0.211 \pm_{0.018}^{0.017}$ & $0.36 \pm 0.02$ \\
\hline LP 55-41 & - & $<0.018$ & $0.008 \pm 0.002$ & $<0.019$ & $<0.034$ & $0.014 \pm 0.004$ & - & $<0.003$ \\
\hline HIP 17695 & $110 \pm_{3}^{2}$ & $4.50 \pm_{0.30}^{0.29}$ & $2.98 \pm 0.14$ & $2.44 \pm_{0.38}^{0.41}$ & $8.88 \pm_{0.39}^{0.40}$ & $1.74 \pm 0.11$ & $1.82 \pm_{0.18}^{0.19}$ & $2.14 \pm_{0.14}^{0.15}$ \\
\hline LP 247-13 & $442 \pm_{103}^{374}$ & $2.04 \pm 0.13$ & $1.77 \pm 0.07$ & $1.48 \pm 0.081$ & $3.57 \pm 0.11$ & $0.918 \pm_{0.052}^{0.056}$ & $0.731 \pm_{0.040}^{0.041}$ & $0.60 \pm 0.04$ \\
\hline GJ 49 & $249 \pm_{93}^{58}$ & $1.38 \pm_{0.07}^{0.08}$ & $1.84 \pm 0.09$ & $0.686 \pm 0.035$ & $2.28 \pm 0.07$ & $0.674 \pm_{0.031}^{0.032}$ & $0.550 \pm_{0.013}^{0.021}$ & $0.47 \pm 0.02$ \\
\hline GJ 410 & $158 \pm_{53}^{163}$ & $2.54 \pm 0.17$ & $2.68 \pm 0.09$ & $0.920 \pm 0.099$ & $2.99 \pm_{0.16}^{0.17}$ & $0.609 \pm 0.051$ & $0.876 \pm_{0.074}^{0.072}$ & $1.06 \pm 0.06$ \\
\hline CD-35 2722 & $25.0 \pm_{1.3}^{2.2}$ & $3.85 \pm 0.18$ & $2.90 \pm_{0.11}^{0.12}$ & $1.74 \pm 0.09$ & $4.49 \pm 0.12$ & $0.90 \pm 0.05$ & $1.32 \pm 0.06$ & $1.29 \pm 0.05$ \\
\hline HIP 23309 & $54.0 \pm_{1.9}^{2.0}$ & $6.60 \pm_{0.28}^{0.29}$ & $3.82 \pm 0.09$ & $2.23 \pm_{0.12}^{0.13}$ & $6.87 \pm 0.19$ & $1.54 \pm 0.06$ & $2.01 \pm_{0.08}^{0.09}$ & $2.42 \pm 0.08$ \\
\hline
\end{tabular}

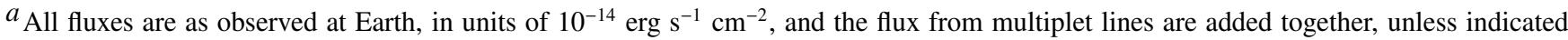
otherwise. Uncertainties reflect the central $68 \%$ confidence interval about the median.

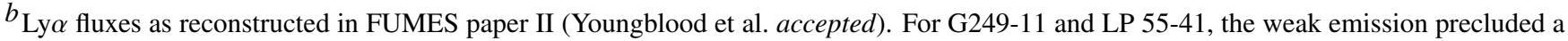
confident fit.

${ }^{c}$ For HIP112312 and HIP17695 with E140M data we report the flux of just the $\lambda 1335 \AA$ line. For the rest of the sample with G140L data, we report the total flux of the blended doublet including ISM absorption. We estimate that these measurements should be corrected upward by $\sim 7 \%$ to account for the influence of the ISM (see Section 3.2).

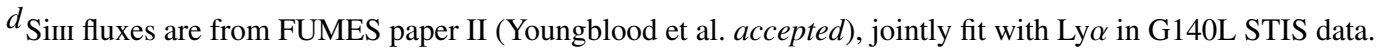

Table 6. FUV Continuum Measurements

\begin{tabular}{lcc}
\hline \multicolumn{1}{c}{ Name } & $\begin{array}{c}\text { Obs Flux } \\
\left(\mathrm{erg} \mathrm{s}^{-1} \mathrm{~cm}^{-2} \AA^{-1}\right)\end{array}$ & S/N \\
\hline GJ 4334 & $5.5 \pm 0.3 \times 10^{-17}$ & 18 \\
GJ 49 & $7.4 \pm 0.3 \times 10^{-17}$ & 25 \\
HIP 112312 $a$ & $5.8 \pm 0.8 \times 10^{-16}$ & 7.25 \\
LP 247-13 & $1.7 \pm 0.05 \times 10^{-16}$ & 34 \\
HIP 17695 $a$ & $3.3 \pm 0.4 \times 10^{-16}$ & 8.25 \\
HIP 23309 & $3.10 \pm 0.09 \times 10^{-16}$ & 34 \\
CD-35 2722 & $1.91 \pm 0.05 \times 10^{-16}$ & 38 \\
GJ 410 & $1.3 \pm 0.1 \times 10^{-16}$ & 13 \\
LP 55-41 & $0.06 \pm 1 \times 10^{-18}$ & - \\
G 249-11 & $5 \pm 6 \times 10^{-19}$ & - \\
\hline
\end{tabular}

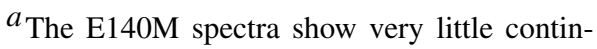
uum if any, leading to likely poorly characterized uncertainties, and thus these values should be taken with caution.

significant, revealing no clear continuum shape, when adding up the emissions across all of the narrow passbands of the spectra the overall continuum emission is detectable. For the quoted uncertainties of Table 6, we propagated the data uncertainty through the continuum flux summation.
Our results are broadly consistent with those of Loyd et al. (2016) accounting for the distance to each target; however, with this broader sample of stars we see evidence for a wide array of continuum levels. Higher signal-to-noise spectra are needed to verify the detection of these average continua and determine what defines their flux levels and spectral composition.

\subsection{Line-Line Correlations}

Our FUV emission line measurements are diagnostic of the stellar upper atmospheres and how they change with magnetic heating. Because they form in similar regions, the line strengths are strongly correlated, as has been well illustrated in the literature (e..g, Youngblood et al. 2017). We build on these results by expanding the available UV samples using our new measurements with the FUMES targets. In addition to the FUMES data we also analyzed the MgII NUV data for the literature sample, as representative of an additional atmospheric layer and spectral region of interest.

Since CIv is a bright and readily accessible emission feature, we show in Figures 5-6 the correlation of each of the emission lines of Table 5 against CIv. We plot the luminosities of each feature as two-dimensional error ellipses $(2 \sigma$ contours) representative of the bivariate error distributions for the luminosities of each line pair. We further discuss the difference in undertaking this analysis in luminosity as compared to surface flux in Appendix A. Since each luminosity depends on the known parallax and its uncertainty, the 

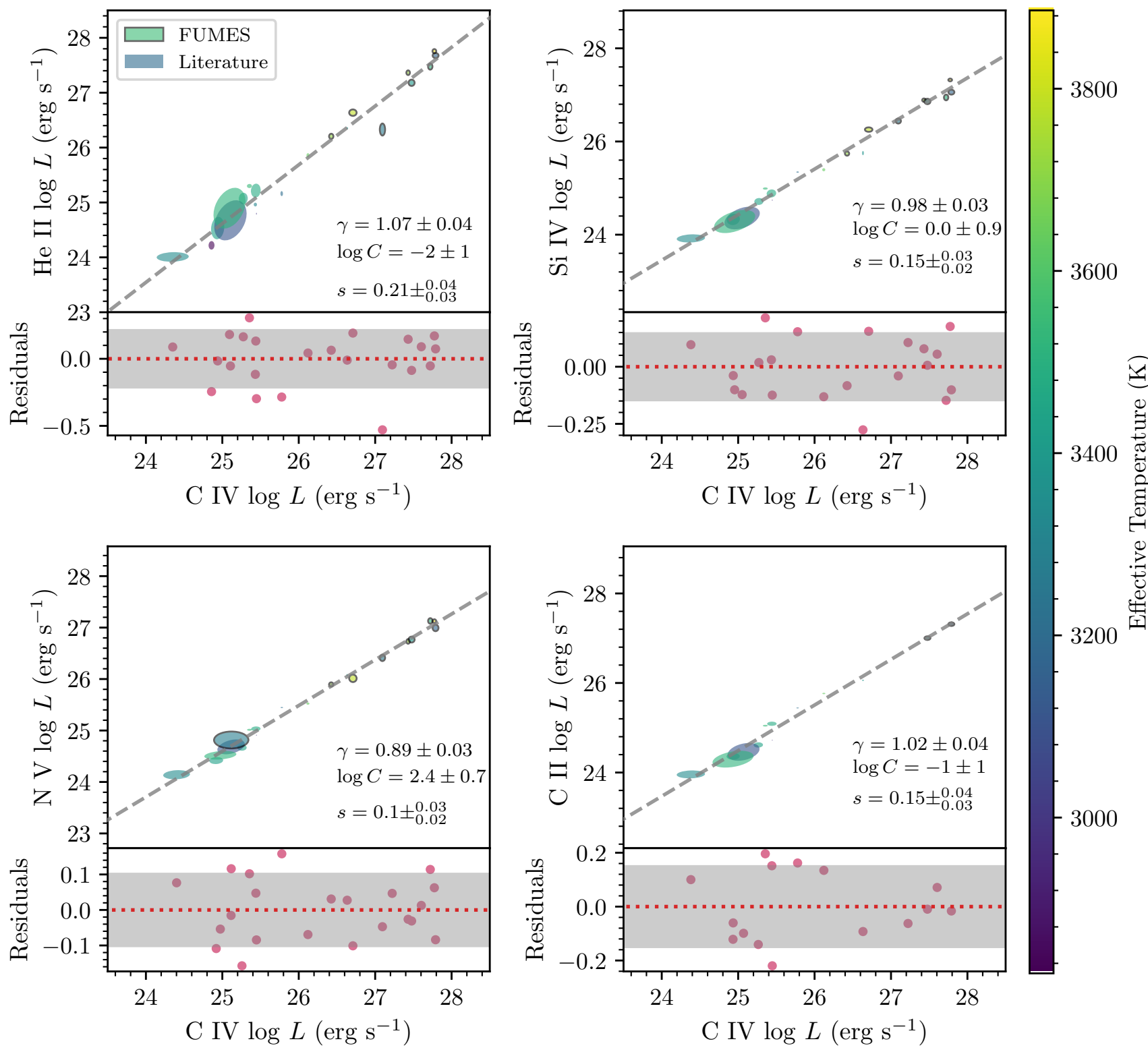

Figure 5. Our FUV emission line correlations for the combined FUMES (black outline) and literature samples show tight power-law fits, $L_{y}=C L_{\text {CIV }}^{\gamma}$, with scatter $s$. Each star is shown as a representative error ellipse corresponding to a $2 \sigma$ confidence level. Dashed lines show the individual best fit relations for each line-pair. Any FUV line can be used to predict any other emission line in quiescence. The lower panel of each plot shows the central residuals from the power-law fit with the intrinsic scatter $s$ shaded in gray.

luminosity determinations have correlated uncertainties, revealed by diagonally oriented ellipses. This correlation is more prominent when the parallax uncertainty dominates the luminosity measurements. Figures 5 and 6 shows these data with the filled shading of each shape indicating the effective temperature of the stars. The emission fluxes are not primarily determined by the stellar effective temperature, as both warmer and cooler objects in the FUMES and literature samples show high and low FUV luminosities, but instead by each object's activity regime, as will be discussed in Section 4.
The correlations shown in Figures 5-6 further demonstrate that the CIV strength can be used to predict the quiescent emission of any of the other prominent transition region emission lines across four orders of magnitude in luminosity. To these data, we fit a power-law relation using Civ as the predictor variable, within a Bayesian framework (Kelly 2007), accounting for uncertainties in both dimensions and incorporating an intrinsic scatter at fixed Civ luminosity. We defined the regression model for the line luminosities, $L_{y}$, as

$$
\log L_{y, i} \mid \gamma, C, s \sim \mathcal{N}_{i}\left(\gamma \log L_{x, i}+\log C, s\right),
$$



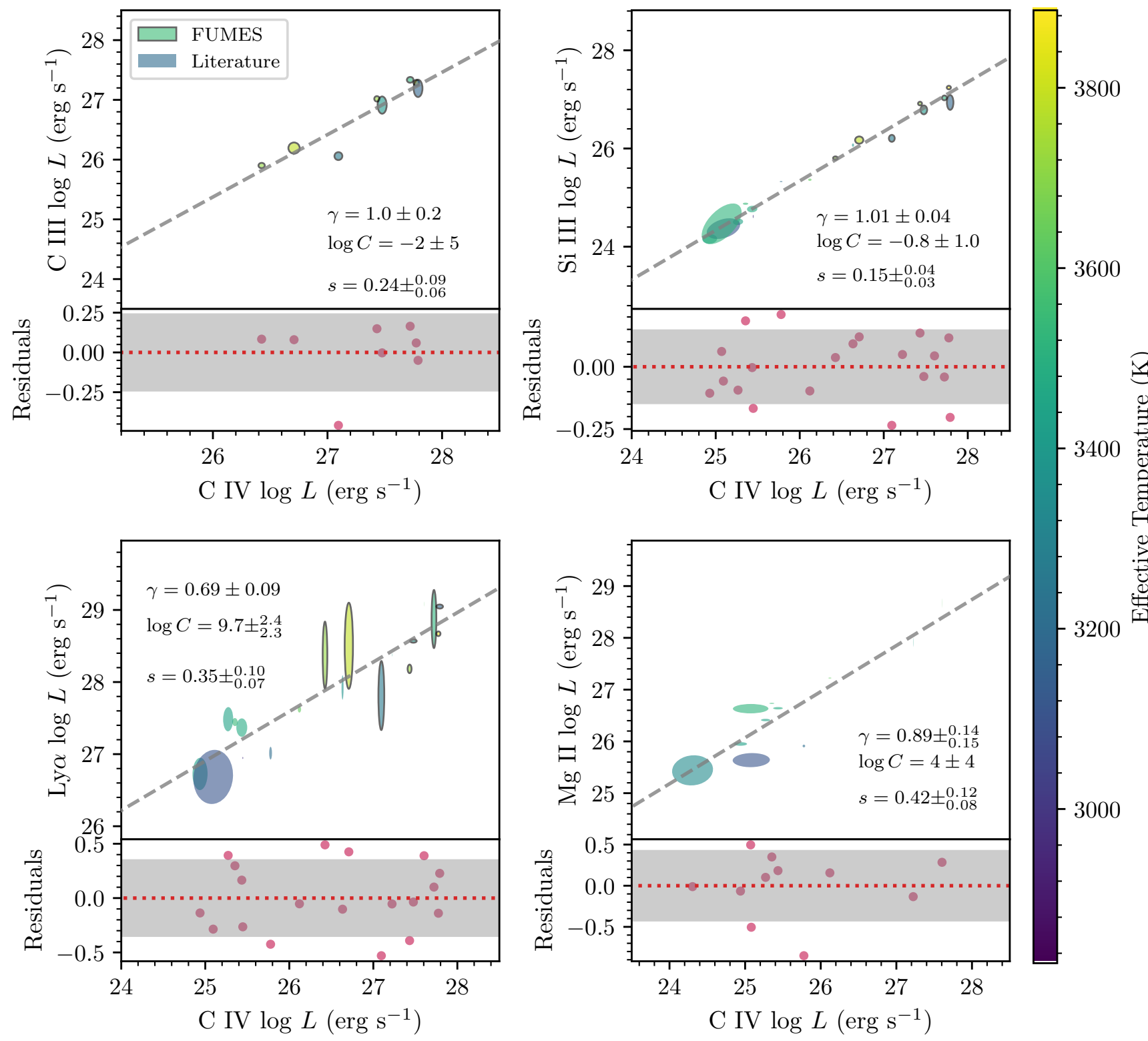

Figure 6. Continued from Figure 5. The correlation for the NUV MgII line shows greater scatter than any of the FUV relations.

where $x$ corresponds to Civ and $y$ any of the other lines (e.g., $\mathrm{Nv}$, Sirv etc.), $\mathcal{N}(\mu, \sigma)$ denotes a Normal distribution of mean $\mu$ and standard deviation $\sigma, i$ denotes each star in the dataset, and $s$ is the intrinsic scatter of the power-law relation in log$\log$ space. ${ }^{12}$, The $s$ parameter is fit along with the power-law model, with its marginalized posterior distribution describing the set of intrinsic scatters consistent with the uncertainty of the data, and the posterior distributions on $\gamma$ and $C$. The peak of that distribution is our best estimate of the representative

\footnotetext{
12 The notation of Equation 1, e.g., $z \mid u, w \sim \mathcal{N}(u, w)$, indicates that the random variable $z$ conditioned on $u$ and $w$ follows the probability density function defined by $\mathcal{N}$.
}

intrinsic scatter in the line-line correlations. As an example, a scatter value, $s=0.1$, would suggest an individual line luminosity could be predicted to within $\sim 25 \%$, corresponding to the central $68 \%$ confidence interval, with a known Civ luminosity.

The probability distribution for each line luminosity pair were defined, in the case of FUMES targets, by their joint distribution created from the sampled line flux posteriors of the model fits (see Section 3.2) combined with the parallax measurement and its uncertainty. For literature data, we assumed Gaussian distributions for the line fluxes and combined with the parallax measurements to determine the joint luminosity distributions at each datum pair. The likelihood is defined by the product across the sample of stars with Equation 1 . 
Table 7. Line Correlations with $\mathrm{CIV}^{a}$

\begin{tabular}{lccccc}
\hline \hline Line & $\log T_{\mathrm{f}}$ & $N_{\text {samp }}$ & $\gamma$ & $\log C$ & $s$ \\
\hline MgII & 4.5 & 11 & $0.89 \pm_{0.15}^{0.14}$ & $4 \pm 4$ & $0.42 \pm_{0.08}^{0.12}$ \\
Ly $\alpha$ & 4.5 & 11 & $0.69 \pm 0.09$ & $9.7 \pm_{2.3}^{2.4}$ & $0.35 \pm_{0.07}^{0.10}$ \\
CII & 4.5 & 15 & $1.02 \pm 0.04$ & $-1 \pm 1$ & $0.15 \pm_{0.03}^{0.04}$ \\
SiIII & 4.7 & 20 & $1.01 \pm 0.04$ & $-0.8 \pm 1.0$ & $0.15 \pm_{0.03}^{0.04}$ \\
CIII & 4.8 & 8 & $1.0 \pm 0.2$ & $-2 \pm 5$ & $0.24 \pm_{0.06}^{0.09}$ \\
HeII & 4.9 & 23 & $1.07 \pm 0.04$ & $-2 \pm 1$ & $0.21 \pm_{0.03}^{0.04}$ \\
SiIV & 4.9 & 21 & $0.98 \pm 0.03$ & $0.0 \pm 0.9$ & $0.15 \pm_{0.02}^{0.03}$ \\
Nv & 5.2 & 22 & $0.89 \pm 0.03$ & $2.4 \pm 0.7$ & $0.10 \pm_{0.02}^{0.03}$ \\
\hline
\end{tabular}

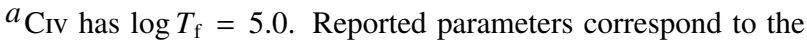
median of the marginalized posterior distributions with uncertainties indicating the central $68 \%$ confidence interval.

In log-log space this is a linear model and we used a uniform prior on $\log C$, the offset, a Cauchy distribution for the powerlaw exponent, $\gamma,{ }^{13}$ and a Half-Cauchy distribution with unity shape parameter for the scatter, $s$. These priors are minimally informative, and only marginally affect the posterior distributions in $\gamma, s$, and $C$, while improving Monte Carlo convergence efficiency. An example of the joint posterior distributions for these fits is shown in Figure 7. The result of these fits are indicated by the dashed lines in Figures 5-6, and we tabulate the fit parameters in Table 7.

The line pair with the smallest intrinsic scatter is Civ-Nv ( $\sim 0.1$ dex). This is unsurprising as these two lines are optically thin and form at similar temperatures high in the transition region. We thus considered whether there may be a trend in intrinsic scatter relative to line formation temperature (see Table 7). The data do not reveal any suggestive trends although MgII and Ly $\alpha$ show the greatest scatter values, and correspond to the lowest formation temperature lines that we studied. On the contrary, $\mathrm{C}_{\text {II }}$ forms at similar temperatures to MgII and shows as small a scatter as the hotter Sirv transition region line. The larger scatter for MgII relation could be due to unaccounted for uncertainty associated with the ISM correction needed for the MgII flux estimates (Youngblood et al. 2016). This may also affect the $\operatorname{Ly} \alpha$ correlation; however, the formation of $\operatorname{Ly} \alpha$, its central profile, and its broad emission (Youngblood et al. accepted) is likely more complicated (Peacock et al. 2019). Predicting that emission from the transition region Civ luminosity thus may not account for all of the processes impacting the $\operatorname{Ly} \alpha$ flux.

\footnotetext{
${ }^{13}$ In practice we used a uniform distribution in the angle, $\theta$, which corresponds to the angle the line in log-log space makes with the horizontal, and set $\gamma=\tan \theta$.
}

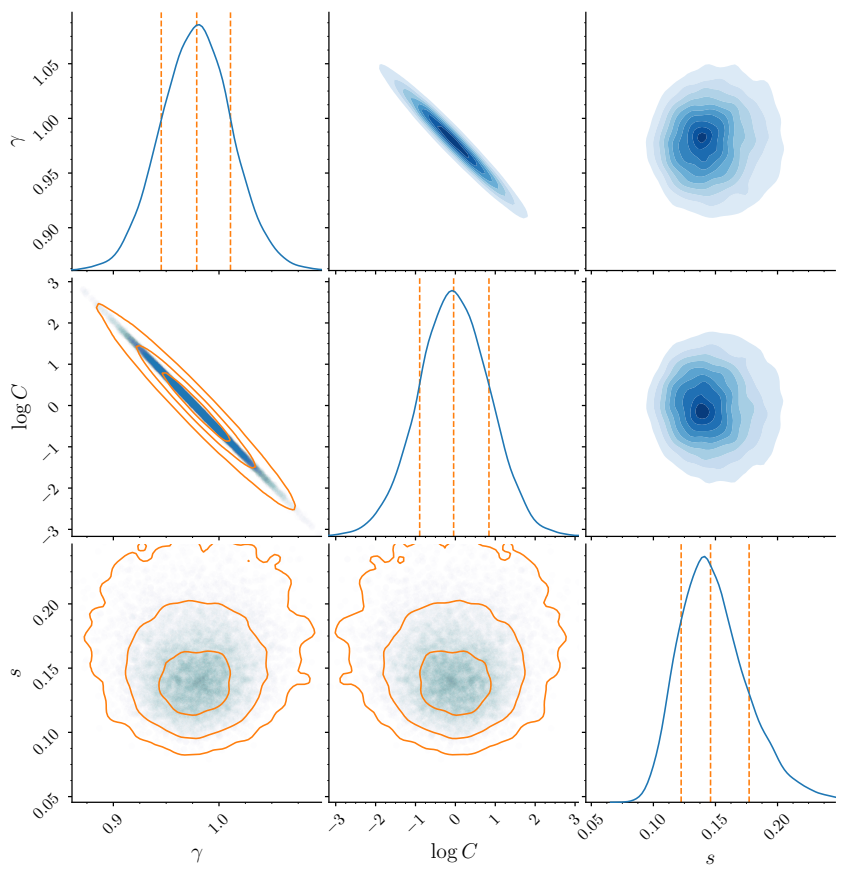

Figure 7. The joint posterior distributions for our typical power-law fits to the line-line correlations (see Section 3.4) illustrate how samples for the power-law slope, $\gamma$, and constant, $\log C$, are highly correlated. The lines in the lower left corner show the $1 \sigma, 2 \sigma$ and $3 \sigma$ contours on top of semi-transparent points from our MCMC sampling. The diagonal shows the marginalized distributions for each property with the distribution visualized from kernel density estimation. Along the diagonal the dashed lines indicate the median and middle $68 \%$ confidence intervals. The upper right corner visualizes the detailed shape of the joint posteriors through kernel density estimation, showing well defined peaks.

The rest of the line-line correlations show consistent scatters of 0.1-0.2 dex at fixed Civ luminosity. These measurements represent the intrinsic scatter of FUV line emission across the population, as all of the observations were either taken simultaneously or closely spaced in time (France et al. 2016). If this is indeed due to intrinsic variation, it may define the limit to which FUV features can be used to predict one another. Additionally, this intrinsic variation may differ between the 'inactive' and 'active' subsamples, however, our samples in each are not large enough to fully investigate.

\section{ROTATION-ACTIVITY RELATION}

\subsection{Regression Model Fitting}

Canonical rotation-activity correlations with optical and X-ray emission (e.g., Pizzolato et al. 2003; Wright et al. 2011; Astudillo-Defru et al. 2017; Newton et al. 2017) have illustrated a strong rotational dependence typically characterized by a power-law distribution, and a saturated regime of activity for the fastest rotators. Since Noyes et al. (1984), these relationships have been used to investigate the inter- 
play between the internal convective motions and differential stellar rotation within an $\alpha-\Omega$ magnetic dynamo, thought to operate in stars with partly convective interiors (e.g., Montesinos et al. 2001; Browning et al. 2006). Mediated by the tachocline interface between the radiative core and convective envelope, the dynamo sustains the magnetic field through cyclic regeneration of toroidal and poloidal field. Theory suggests that the magnetic fields, and hence the resulting activity tracers should thus be mediated by the Rossby number, $R o=P / \tau_{c}$, the ratio between the rotational period and the convective turn over time, which characterizes the timescales for bulk motions of internal convection. Recent results have further extended these studies across the boundary between fully convective and partly convective interiors (Newton et al. 2017; Wright et al. 2018) with M dwarf samples, illustrating a continued rotational dependence for the activity tracers, despite the need for alternative dynamos for the lowest mass stars, which lack that tachocline interface.

With the FUMES sample, we can now probe these same mechanisms with new indicators of the magnetic activity in the FUV. To our emission line data, we fit a broken powerlaw as a function of $R o$,

$$
L_{y} / L_{\mathrm{bol}}= \begin{cases}\left(L_{y} / L_{\mathrm{bol}}\right)_{\mathrm{sat}}, & R o \leq R o_{c} \\ A(R o)^{\eta}, & R o>R o_{c},\end{cases}
$$

where $\left(L_{y} / L_{\mathrm{bol}}\right)_{\text {sat }}$ denotes the strength of emission line $y$, in the saturated regime relative to the star's bolometric luminosity, $\eta$ indicates the slope of the unsaturated regime, and $R o_{c}$ is the critical Rossby number at which the activity transitions between regimes, with $A \equiv\left(L_{y} / L_{\mathrm{bol}}\right)_{\mathrm{sat}}\left(R o_{c}\right)^{-\eta}$ to ensure continuity. To compute the Rossby number, we use the known rotation periods (see Table 2), and the empirical calibration for the convective turn overtime, $\tau_{c}$, as a function of mass from Wright et al. (2018). We discuss systematic effects in this choice of calibration in Appendix B. Our segmented regression fit further includes a scatter term, $\sigma_{L}$, describing intrinsic dispersion in the observed luminosities at a fixed Rossby number, such that

$$
\log \left(L_{y} / L_{\mathrm{bol}}\right)_{i} \mid\{\beta\} \sim \mathcal{N}\left(\log \left[L_{y} / L_{\mathrm{bol}}\left(R o_{i}\right)\right], \sigma_{L}\right),
$$

where the index $i$ indicates the data for each star, and $\{\beta\}=$ $\left\{R o_{c},\left(L_{y} / L_{\mathrm{bol}}\right)_{\mathrm{sat}}, \eta, \sigma_{L}\right\}$ is the set of four model parameters. Within this Bayesian model we employ as priors uniform distributions for $\log \left(L_{y} / L_{\mathrm{bol}}\right)_{\mathrm{sat}}$ and $R o_{c}$, a zero centered Cauchy distribution with unity shape parameter for the slope $\eta$ (see footnote 13), and Half-Cauchy distribution with unity shape parameter for $\sigma_{L}$.

Our fitting process using PyMC 3 accounts for random uncertainty in both dimensions (Kelly 2007), and possible error correlations between the assumed mass, and bolometric luminosity using the sampled posterior distributions from the
Table 8. Rotation-Activity Correlations $a$

\begin{tabular}{lccccc}
\hline \hline Line & $N_{\text {samp }}$ & $\eta$ & $R o_{c}$ & $\log \left(L_{\text {line }} / L_{\text {bol }}\right)_{\text {sat }}$ & $\sigma_{L}$ \\
\hline Ly $\alpha$ & 19 & $-1.26 \pm_{0.58}^{0.41}$ & $0.21 \pm_{0.11}^{0.16}$ & $-3.58 \pm_{0.15}^{0.16}$ & $0.32 \pm_{0.07}^{0.10}$ \\
MgII & 12 & $-1.86 \pm_{0.55}^{0.41}$ & $0.20 \pm_{0.08}^{0.11}$ & $-3.99 \pm_{0.15}^{0.14}$ & $0.23 \pm_{0.07}^{0.10}$ \\
CII & 15 & $-2.38 \pm_{0.77}^{0.64}$ & $0.24 \pm_{0.11}^{0.12}$ & $-5.25 \pm 0.16$ & $0.33 \pm_{0.07}^{0.10}$ \\
SiIII & 20 & $-2.08 \pm_{0.46}^{0.41}$ & $0.20 \pm 0.07$ & $-5.37 \pm 0.13$ & $0.32 \pm_{0.06}^{0.08}$ \\
HeII & 24 & $-2.19 \pm_{0.33}^{0.30}$ & $0.19 \pm 0.05$ & $-4.92 \pm 0.11$ & $0.27 \pm_{0.04}^{0.06}$ \\
SiIV & 21 & $-2.32 \pm_{0.44}^{0.42}$ & $0.24 \pm 0.07$ & $-5.38 \pm 0.14$ & $0.35 \pm_{0.06}^{0.08}$ \\
CIV & 24 & $-2.02 \pm_{0.41}^{0.39}$ & $0.18 \pm_{0.06}^{0.07}$ & $-4.72 \pm 0.15$ & $0.37 \pm_{0.06}^{0.08}$ \\
Nv & 23 & $-1.84 \pm_{0.40}^{0.37}$ & $0.19 \pm 0.07$ & $-5.37 \pm_{0.13}^{0.14}$ & $0.33 \pm_{0.06}^{0.07}$ \\
\hline
\end{tabular}

$a_{\text {Reported parameters correspond to the median of the marginalized poste- }}$ rior distribution with uncertainties indicating the central $68 \%$ confidence interval.

work of Pineda et al. (in prep). The error correlations between $M$ and $L_{\mathrm{bol}}$ are generally small for the field objects, but can be significant for the young stars (see Pineda et al. in prep). We also included the scatter in the convective turnover time calibration from Wright et al. (2018) of 0.055 dex in $\log \tau_{c}$ at fixed mass. When using the literature periods, we further incorporated the quoted uncertainties in those measurements if available or used a $10 \%$ Gaussian uncertainty if not, see Table 2 (private communication, Newton, E.). Except for the $\operatorname{Ly} \alpha$ measurements of some stars, the random uncertainties in the rotation-activity data are typically dominated by the error on the Rossby number driven by the scatter in the $\tau_{c}$ calibration. This careful accounting of the known sources of random error enabled us to carefully examine systematic effects with this fitting (see Appendix B).

We applied these methods to eight UV emission lines, $\operatorname{Ly} \alpha$, MgII, CII, SiIII, HeII, Sirv, Civ, and Nv, for which the samples permitted a detailed rotation-activity fit. We show the best parameter results in Table 8 from the marginalized posteriors, with the fit solutions plotted in Figure 8. We also show an example of the joint posterior distributions for this fitting in Figure 9, illustrating how the critical Rossby number and unsaturated regime slope are generally correlated in our rotation-activity parameterization of Equation 2.

Because this regression analysis relies on stellar mass estimates for determining $R o$, it may be potentially biased by our choice for the young stars to utilize the magnetic model based masses, which are larger than the non-magnetic model masses (see Pineda et al. in prep), with a correspondingly larger implied Ro. This concern applies only to the five young stars in our sample (see Table 2). However, of the five, only AU Mic (Ro = 0.11-0.17) appears to be near the transition between saturated and unsaturated regimes. HIP 112312, HIP 17695, and CD-35 2722 have Ro val- 

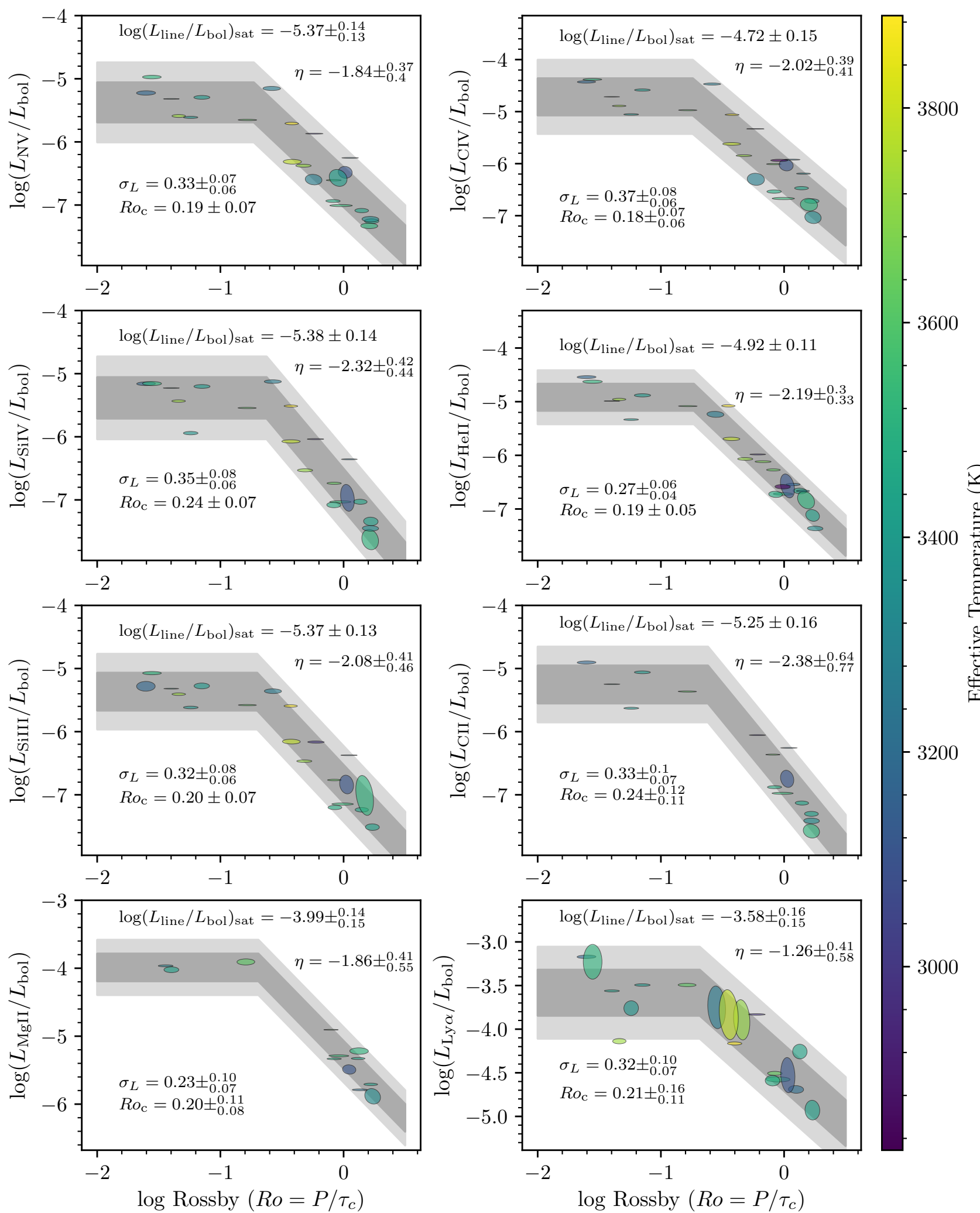

Figure 8. Rotation-activity correlations are prominent across all of the UV emission features analyzed in this work. Individual data points are represented by $1 \sigma$ error ellipses, shaded to indicate each star's effective temperature. The best fit broken power law models (see Section 4 ) are shown in gray illustrating the $1 \sigma$ (dark gray) and $2 \sigma$ (light gray) scatters. 


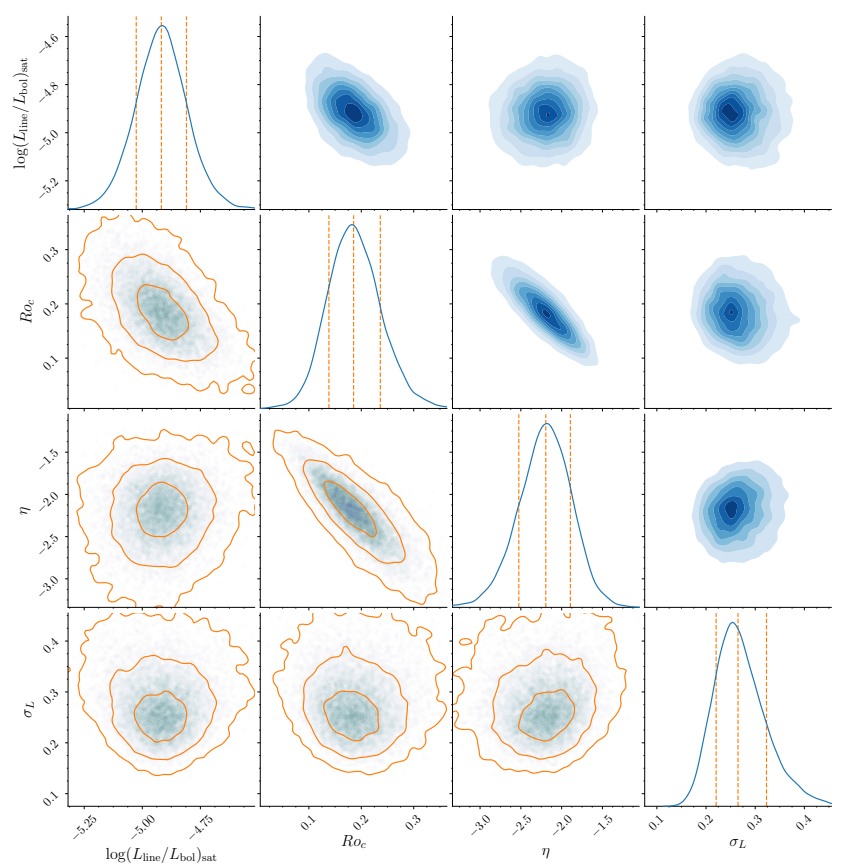

Figure 9. The HeI joint posterior distributions (displayed as in Figure 7) for the broken power-law fit (see Section 4) of the rotationactivity relation illustrate the correlation of the slope $(\eta)$, and critical Rossby number $\left(R o_{c}\right)$. The posterior results for fits to the other features are qualitatively identical, with results shown in Table 8.

ues well below 0.1 regardless of the model choice for the mass estimate, and HIP 23309 has a $R o=0.32-0.39$, larger than the literature values of $R o_{c} \sim 0.15-0.20$ (Newton et al. 2017; Wright et al. 2018). Because horizontal systematics within the saturated regime have no influence on the rotationactivity fits, and only one of the stars in a sample of $\gtrsim 20$ targets per emission line might be affecting the analysis, we consider this systematic effect in young star masses to minimally impact our results. Furthermore, these young stars do not appear to be outliers in our rotation-activity plots (Figure 8). Although the difference in Ro is small, only the result for MgII is likely to be impacted by the mass choice for AU Mic, because of the limited target sample for that line. Correspondingly, the rotation-activity fit parameters for MgII have larger uncertainties.

We further consider the quality of our fits by examining the residuals, as illustrated in Figure 10. The data residuals are consistent with being normally distributed about the best median rotation-activity relation, and show no apparent dependence on stellar mass. There may be some hints of an excess of data points below the median fit at large Rossby numbers $(\sim 1)$, but this sample is too small to be conclusive in this regard. This may be more evident in literature studies (e.g., Newton et al. 2017), which would indicate that at slow

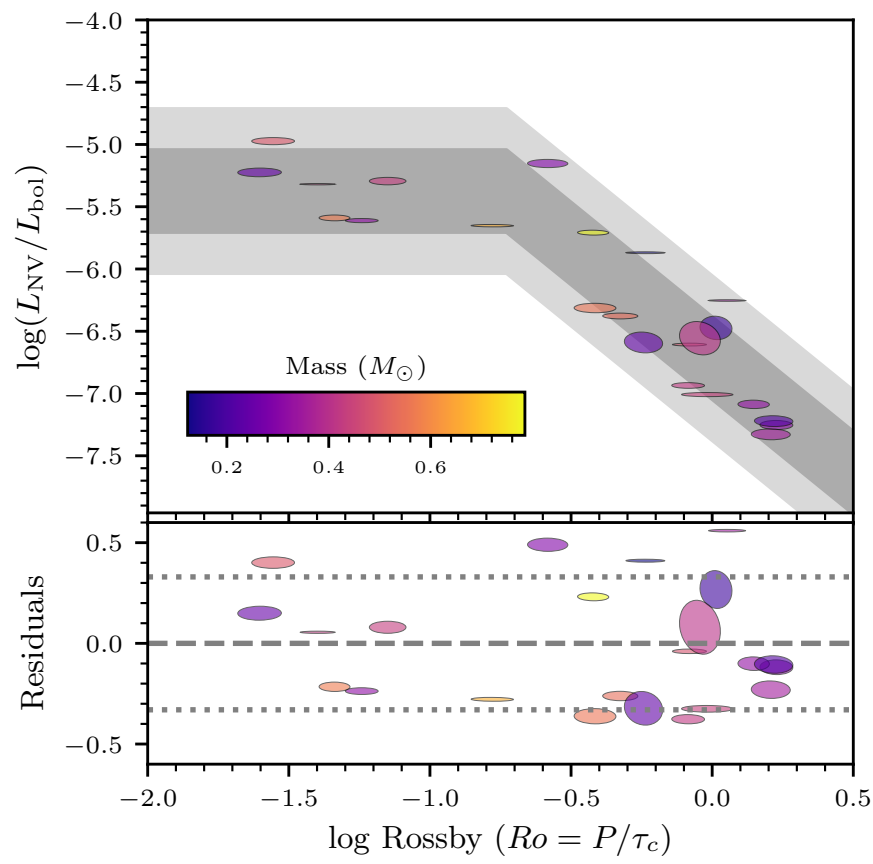

Figure 10. Our rotation-activity model provides a good fit across our entire sample for each emission line, with the results for $\mathrm{Nv}$ shown here again as an example, but now with error ellipses shaded according to stellar mass. The residuals are consistent with a normal distribution, and show no significant correlation with mass (Pearson coefficient of -0.25). The shaded region of top panel are as in Figure 8 , with dotted-line in lower panel indicating that same scatter value, $\sigma_{L}$.

rotation periods the single power-law may be overestimating the magnetic activity.

With these considerations, for all of the emission lines, we deem the broken power-law to accurately describe the rotation dependence of the UV activity indicators across the entire sample, with each line showing slightly different emissions levels in the saturated regime, consistent critical Rossby values of $\sim 0.2$, and a range of slopes in the unsaturated regime spanning -1.3 to -2.4 (see Table 8 ). For our Mdwarf sample the saturation levels of $\log \left(L_{\text {line }} / L_{\mathrm{bol}}\right)_{\text {sat }}$ in the CII, SiIII, Nv, and Sirv lines all exceed those measured by France et al. (2018) for an ensemble of FGKM stars, which were all in the range of -5.5 to -6 . In the saturated activity regime, M-dwarf FUV emissions exceed that of warmer stars relative to bolometric. For the power-law decay with decreasing rotation, all the lines except $\operatorname{Ly} \alpha$ are consistent with a slope of -2 , with $\operatorname{Ly} \alpha$ corresponding to the shallowest slope in the rotation-activity analysis. While the error bar is large, because these data rely on model reconstructions of the emission flux, we consider if those assumptions may be systematically impacting this result.

\subsection{Ly $\alpha$ and Evolving Emission Line Ratios}




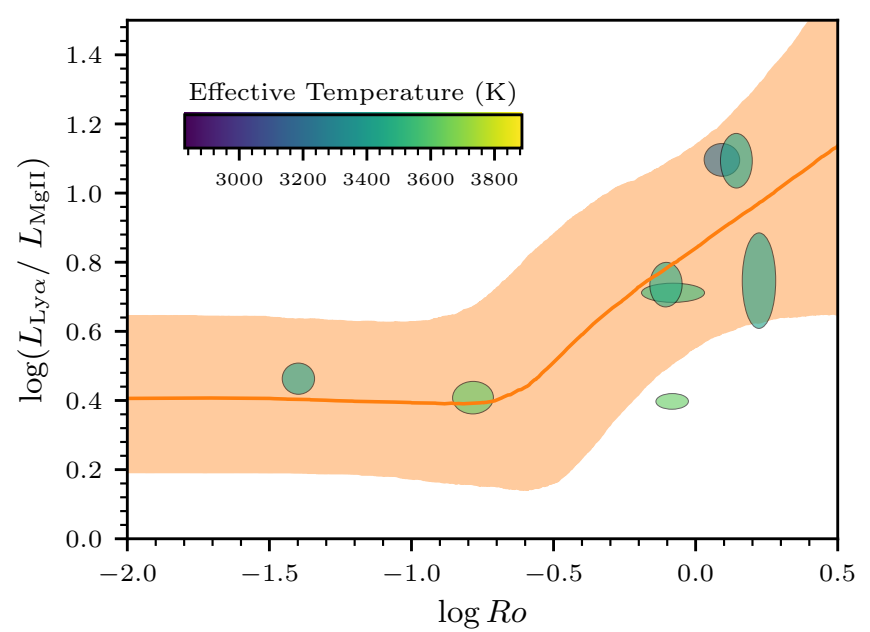

Figure 11. The ratio of $\operatorname{Ly} \alpha$ to $M g I I$ emission, which tracks the FUV/NUV stellar flux ratio, rises with increasing Rossby number over the course of stellar angular momentum evolution. The shaded area denotes the central $68 \%$ confidence region defined by combining the rotation-activity fits to $\mathrm{Ly} \alpha$ and MgII, see Section 4.2.

Our Ly $\alpha$ reconstructions (Youngblood et al. accepted) and those from the literature sample (Youngblood et al. 2016), typically assume a Voigt-like profile for the emission, however, there is some evidence and theory suggesting that the ISM obscured Ly $\alpha$ profile peak may show an absorption selfreversal like that observed in other chromospheric lines (e.g., Linsky et al. 1979; Redfield \& Linsky 2002; Guinan et al. 2016; Youngblood et al. 2016; Peacock et al. 2019). Our Ly $\alpha$ reconstructions do not account for this effect, and we may thus be overestimating the Ly $\alpha$ flux. However, for this effect to impact the fitted slope in the rotation-activity unsaturated regime, the strength of self-reversals would also need to depend on the Rossby number. Theoretical Ly $\alpha$ line profiles from Peacock et al. (2019) for GJ 832, GJ 176, and GJ 436 also show strong core absorption due to non-LTE effects, such that the reconstructed fluxes are greater by a factor of $\sim 2$. A systematic effect at this level or stronger between active and inactive M-dwarfs is needed to explain the shallow $\operatorname{Ly} \alpha$ slope. However, more data is required to validate whether these theoretical profiles match those produced by nature. There is currently no evidence for such a systematic difference in $\operatorname{Ly} \alpha$ core profiles between active and inactive M-dwarfs.

If typical Ly $\alpha$ lines only show weak self-reversals in Mdwarfs as suggested empirically by Guinan et al. (2016) and Bourrier et al. (2017), then the shallow slope could indicate the persistence of Ly $\alpha$ emission at slow rotation rates even as other high-energy features decay more rapidly. As the most prominent emission lines in the FUV and NUV respectively, we consider the evolution of the ratio of Ly $\alpha$ to MgII emissions. We illustrate this in Figure 11, showing the emission ratio as a function of Rossy number. The shaded region denotes the ratio of the power-law fits in the two lines from Section 4.1 consistent with the uncertainties in the parameter estimates to the $1 \sigma$ level. More data are needed to better refine rotation-activity relationships in both features, but the rise in ratio with angular momentum evolution indicates that the relative strengths of different portions of the highenergy spectrum changes over time. Evolutionary changes in the spectral illumination has implications for the prevalent photochemistry and atmospheric history of any exoplanetary systems orbiting low-mass hosts. For example, stronger FUV relative to NUV emissions may drive a build-up of photochemical ozone in planetary atmospheres (e.g. Gao et al. 2015; Harman et al. 2015). Although a full account of the relative contribution of MgII to the total NUV luminosity is necessary for a complete description, our data suggest that this effect may increases as stars spin-down over time. Furthermore, such an evolutionary effect may be more prominent for early M-dwarfs, and not as significant for late M-dwarfs (Schneider \& Shkolnik 2018).

\subsection{Trends with Formation Temperature}

Our results across all of the lines (Table 8) reveal that most of the best fit slopes are around the canonical value of $-2 \mathrm{im}$ plied by a distributed dynamo model (Noyes et al. 1984) in which dynamo amplification occurs throughout the convective region. Whereas in an interface dynamo like that based on a tachocline there are additional dependencies which lead to deviations away from this canonical slope of -2 (Parker 1993; Charbonneau \& MacGregor 1997; Wright et al. 2011). To compare these results from the different lines, we plot our fit parameters for $\eta$ and $R o_{c}$ as a function of line formation temperature in Figure 12. The UV lines are plotted at the temperatures listed in Table 7, to which we also add the $\mathrm{H} \alpha$ results of Newton et al. (2017) as representative of the chromosphere, and the X-ray results of Wright et al. (2011, 2018) corresponding to the corona using a nominal temperature of $10^{6} \mathrm{~K}$, although X-ray flux contributions extend to higher temperatures. In Figure 12, we show the mean value representative of the transition region, taking the combined posteriors across all the lines in each parameter, yielding medians and central $68 \%$ confidence intervals of $\eta=-2.02 \pm_{0.53}^{0.55}$ and $R o_{c}=0.20 \pm_{0.07}^{0.09}$. Our mean result is consistent to within the uncertainties with both the chromospheric and coronal results in the literature.

However, there may be a trend in the unsaturated rotationactivity slope as a function of formation temperature. While this is largely a consequence of different values for the $\mathrm{H} \alpha$ and X-ray results, our UV data fall directly in between. As stars spin down in the unsaturated regime, the coronal X-ray emissions appear to decline rapidly with deeper atmospheric layers showing a slower decay in their magnetic activity. The 

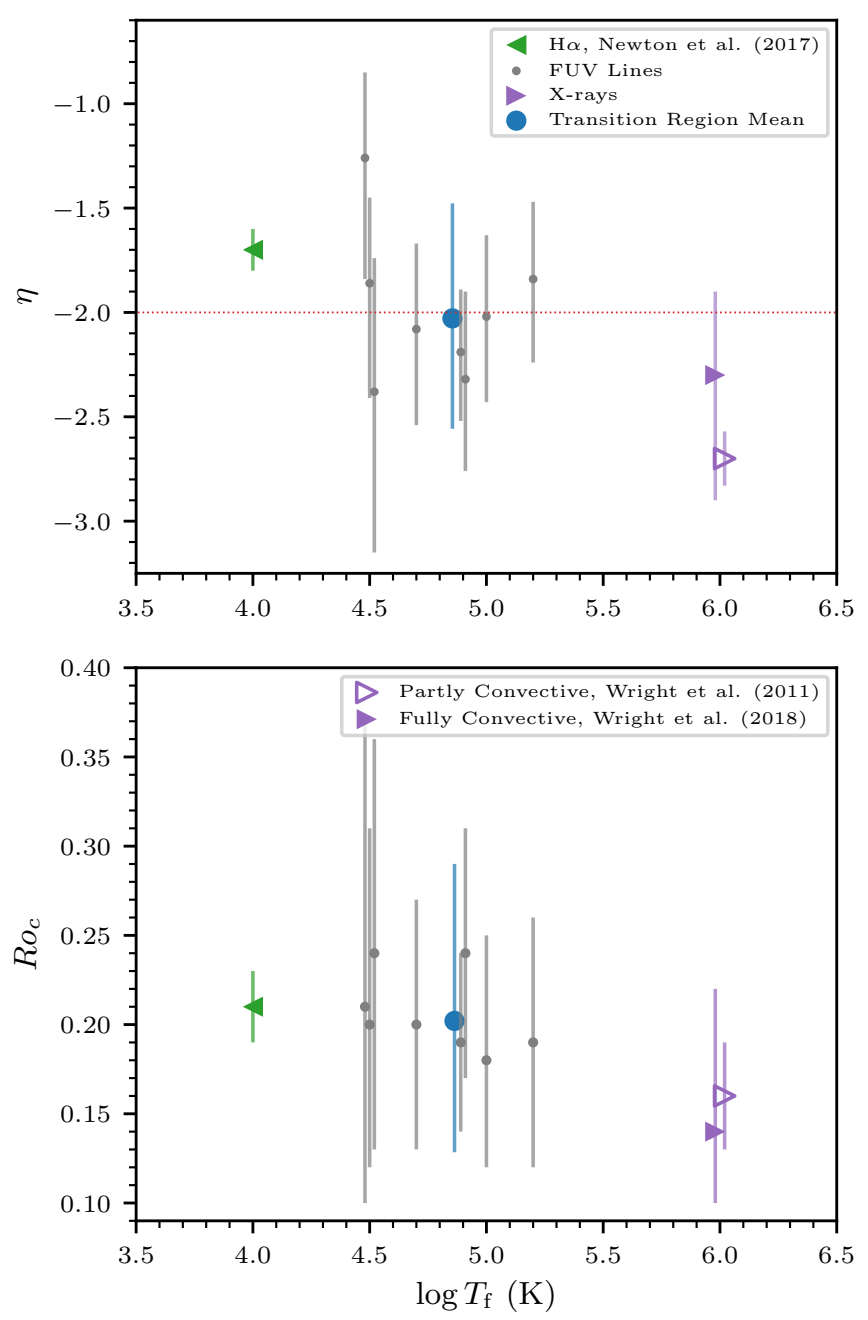

Figure 12. Our new results for the transition region rotation-activity relationships, unsaturated slope, $\eta$ (Top), and critical Rossby number, $R o_{c}$ (bottom), enable a comparison across features probing distinct regions of the stellar upper atmosphere. As activity declines with slower rotation, the layers of the atmosphere from the chromosphere to the corona may respond differently, see Section 4.3.

onset of activity decline with slower rotation begins first in $\mathrm{X}$-rays, and only after some angular momentum evolution the transition region and chromospheric features also begin to decline, since the best fit $R o_{c}$ are greater for the deeper layers.

If these trends hold from analyses of larger UV samples, they will help reveal the role of magnetic heating processes in mediating the relationship between the internal magnetic dynamo and the emission features. While the rotation-activity relationships have been used to constrain stellar dynamos, this connection is indirect. The emission is necessarily a consequence of the non-thermal magnetic heating, and if the different layers exhibit distinct power-law slopes, then this effect of the heating processes needs to be accounted for when making dynamo inferences from rotation-activity relationships. The trend evident in the top panel of Figure 12 for the unsaturated slope suggests that whether the dominant process is Alfvén wave heating or nano-flare reconnection, the decline in non-thermal heating with weaker average field strengths (e.g., Shulyak et al. 2017) as stars spin down takes place from the outer most layers inward. In other words, the magnetic heating processes persist more strongly in deeper atmospheric layers across angular momentum evolution affecting the relative strengths of emission features formed in different layers of the atmosphere.

There are however some important caveats to the comparisons implicit in Figure 12. Between this work and the studies of Newton et al. (2017) and Wright et al. (2011, 2018), the samples are not identical. Our work and Newton et al. (2017) use similar mixed age samples of M-dwarfs, whereas the Xray data of Wright et al. (2018) also includes more massive stars, although normalizing by the bolometric luminosity and examining the rotation relationship in $R o$ is designed to account for these possible differences. More significantly, as we discuss in Appendix B, differences in the Ro calibration can impart systematic effects on the rotation-activity analyses. Newton et al. (2017) used the calibration of Wright et al. (2011), whereas we used that of Wright et al. (2018). Relative to our work the Newton et al. (2017) results for critical Rossby number and unsaturated slope could be systematically greater than if they used the same calibration. The magnitude of this effect however can be small and depends on the stellar sample (see Appendix B). Moreover, there may have been issues with the analyses of Wright et al. (2011) leading to a much steeper power-law slope (Reiners et al. 2014). Nevertheless, it appears that in the chromosphere $\eta$ is likely shallower than -2 , whereas in the corona it is steeper than -2 , with the transition region value from our work right in the middle. Consistent methods and calibrations need to be applied across the different wavebands to discern whether there are indeed rotation-activity trends as a function of where in the stellar atmosphere the emission originates.

\subsection{Alternatives to Rossby Number?}

Potential issues with a choice of calibration can be avoided by circumventing the Rossby number entirely. Reiners et al. (2014) argue for a more general approach in analyzing the rotation-activity relationships of low-mass stars, and conclude that the activity dependence can be well described by a combination of rotation period and radius, specifically $L_{X} / L_{\text {bol }}=k P^{-2} R^{-4}$, where $k$ is a scaling constant. We consider how this relationship may apply to our UV data. In Figure 13, we show the HeII luminosity normalized by stellar bolometric luminosity against the stellar $P^{-2} R^{-4}$. Like the Rossby scaling, Figure 13 illustrates a potentially power-law decay in emission with a scatter of activity at a given ab- 


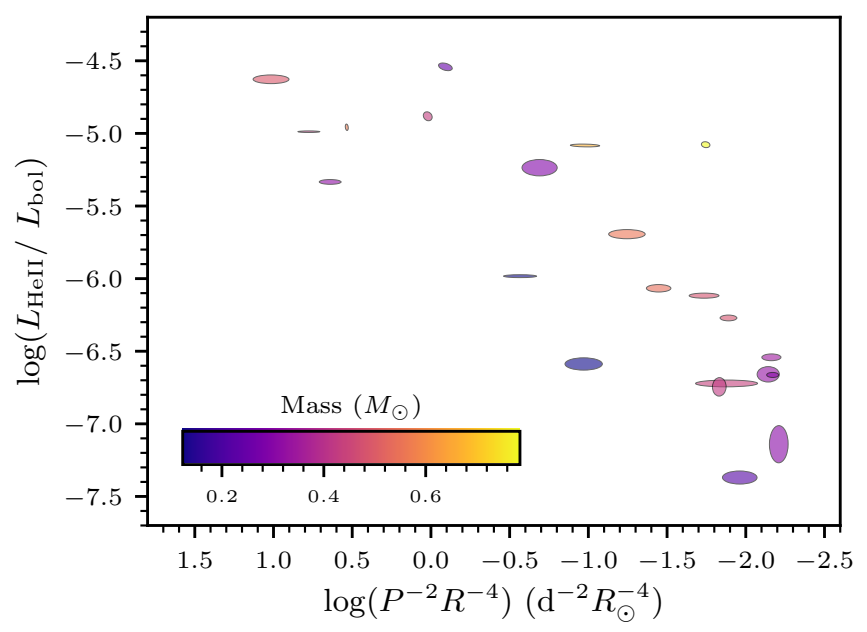

Figure 13. The UV emission of our sample, traced by HeII, plotted as representative $1 \sigma$ ellipses against the rotation period and radius provides an alternative description for the rotation-activity relationship. These data show a possible mass dependence in the activity, see Section 4.4.

scissa. Although our sample is much smaller, there are hints of the mass dependence in activity using this scaling that was illustrated by Newton et al. (2017) with $\mathrm{H} \alpha$ data by which more massive stars preferentially lie to the right of the locus of points in Figure 13. The mass dependence appears to increase the intrinsic scatter at fixed rotation in the activity beyond the $\sim 0.27$ dex scatter evident in our fits to $L_{\mathrm{HeII}} / L_{\text {bol }}$ vs. $R o$.

While it is evident that rotation still plays a key role in the magnetic emission of fully convective stars, an empirical Rossby number may not be the most appropriate scaling to uncover the underlying relationships governing the dynamo action, and possible differences with partly convective stars. It appears to work sufficiently well across the chromosphere, transition region and corona, however, by defining $R o$ specifically to minimize scatter in the rotation-activity diagrams across this full range of stars, this procedure may be masking real differences in the evolution of magnetic activity between partly and fully convective objects (e.g., Magaudda et al. 2020). If these two stellar mass regimes did indeed show distinct rotation-activity correlations, i.e., statistically distinguishable rotational dependencies of their activity decay, the process of defining an empirical Rossby number across both samples would be averaging together these possible differences, since it presupposes that both samples follow the same power-law relation.

\section{TEMPORAL EVOLUTION}

In Section 4, we used the stellar rotation period as a proxy for examining magnetic activity throughout the lifetimes of low-mass stars. However, using gyrochronology (e.g., Barnes 2003), we can also compare our FUV activity indicators directly to stellar ages using literature relations connecting rotation period and age. While this practice is well established for solar-like stars (e.g., Angus et al. 2015; Gallet \& Bouvier 2015), the process is much more difficult for M-dwarfs (e.g., Guinan et al. 2016), which lack a multitude of targets with well determined ages. Nevertheless, Engle \& Guinan (2018) have developed empirical relations for M0-M1 and M2.5-M6 dwarfs to estimate ages from measured rotation periods. Although such gyrochronology relations for M-dwarfs remain ongoing work, we use those results in this paper to provide an approximate indication of the expected behavior across the low-mass star regime.

\subsection{UV Emissions Across Time}

We thus show the evolution of FUV activity with age in Figure 14 , focusing on the $\mathrm{Nv}$ emission feature as representative of all of the other FUV lines (Sections 3.4 and 4). We chose the Nv line for this analysis as it is tightly correlated with CIV, and existing literature relations allow us to also estimate EUV emission from their measurements, see below.

For the five objects with moving group membership (see Table 2), we used the mean group age with error, and the gyrochronology relations for the rest of the sample. To assess the uncertainties on the ages from the gyrochronology calibration, we used the reported uncertainties of the parameters of the best-fit relations (Engle \& Guinan 2018) and the period uncertainties as in Section 4.1, sampling each appropriately, assuming independently Gaussian distributed variables in computing the distribution for the age estimate. ${ }^{14}$ Because the Engle \& Guinan (2018) rotation-age relations are defined by two bins of spectral type ranges, for our sample we used the appropriate relation corresponding to the optical spectral types listed in Table 2. However, some of our objects have spectral types, M1.5-M2, between the defined ranges for the rotation-age relations. For these objects we combined the estimates from both the M0-M1 and M2.5-M6 relations to determine the age estimate and its uncertainty. Three of these stars were rapid rotators without known ages: AD Leo, EV Lac, and LP 247-13. Given the large uncertainty in the gyrochronology ages, especially early on where the rotational evolution has yet to converge, we plot these age estimates as $3 \sigma$ upper limits (triangles in Figure 14).

In Figure 14, we also shade the points according to their effective temperature. Based on these age estimates, Figure 14 illustrates how cooler, later M-dwarfs persist with strong ac-

\footnotetext{
14 Engle \& Guinan (2018) did not report a scatter about their best fit rotationage relations, so we could not incorporate that uncertainty in these age estimates. For the M0-M1 bin, we ignored the uncertainty on their powerlaw exponent, as the results are too sensitive to its value and we cannot properly account for its correlation with the other parameters that would mitigate this effect.
} 


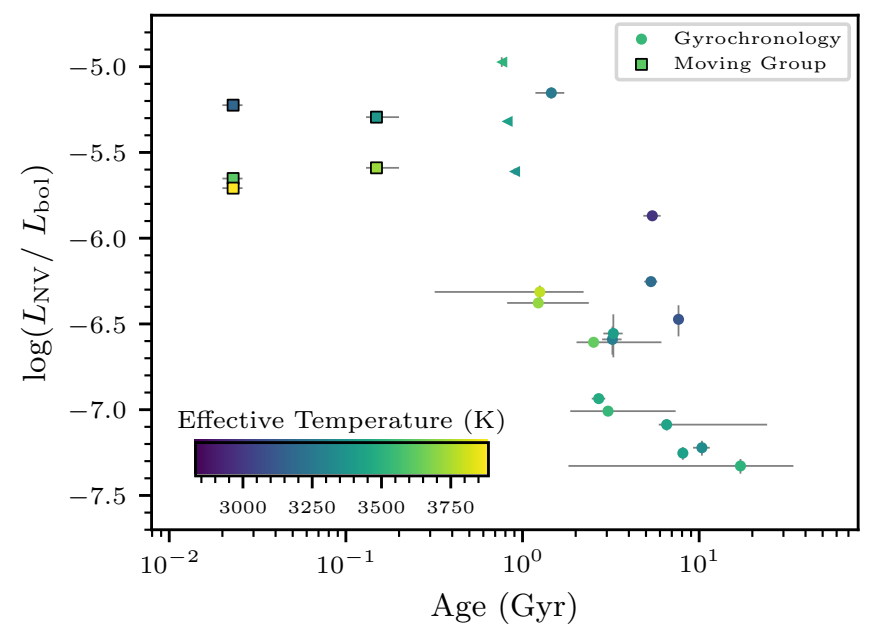

Figure 14. The Nv luminosity of our sample as a function of age illustrates how young M-stars exhibit saturated FUV emissions which last for $\sim 1 \mathrm{Gyr}$, before declining. The cooler M-dwarfs also show strong UV emissions for longer into their lifetimes than warmer objects. Age uncertainties $(1 \sigma)$ are determined from the group age determination in the literature, or from the uncertainties in the gyrochronology relations, with upper limits for the rapid rotation star ages indicated as triangles, see Section 5.

tivity levels for a longer duration of their early lifetimes. Considering the mean level of $\log \left(L_{\mathrm{NV}} / L_{\mathrm{bol}}\right)_{\mathrm{sat}} \sim-5.37$ and 0.33 dex scatter, some of the cooler objects may display near saturation level activity beyond an age of $1 \mathrm{Gyr}$. In contrast, the warmer M-dwarfs, by this age, appear to have declined in their magnetic emissions by an order of magnitude relative to the saturated regime. Using GALEX photometry, Schneider \& Shkolnik (2018) also found this divergence in UV evolution between early and mid-M dwarfs.

Because of its importance to exoplanetary atmospheric heating, we also considered what our FUV evolutionary data imply for the extreme ultraviolet portion of the high-energy spectrum. To estimate the EUV from the FUV data, we used the empirical relation of France et al. (2018) between Nv emission and the blue portion of the EUV passband, EUVb (90-360 $\AA$ ). While not the full EUV spectrum, this is the portion for which available data and relatively low ISM attenuation have permitted any empirical estimates in the low-mass star regime. This EUVb band corresponds to about half of the total EUV energy from M-dwarf stars (Fontenla et al. 2016; France et al. 2018). In Figure 15, we plot this EUV evolution with the same ages as discussed above, and EUVb luminosities determined from the $\mathrm{Nv}$ emission, incorporating the parameter uncertainties and 0.24 dex scatter in the France et al. (2018) relation. For young active M-dwarf stars the EUV luminosity is $\sim 10^{-3.5}$ relative to bolometric, with these emissions lasting for the first several hundreds of Myrs of their lives and perhaps longer.

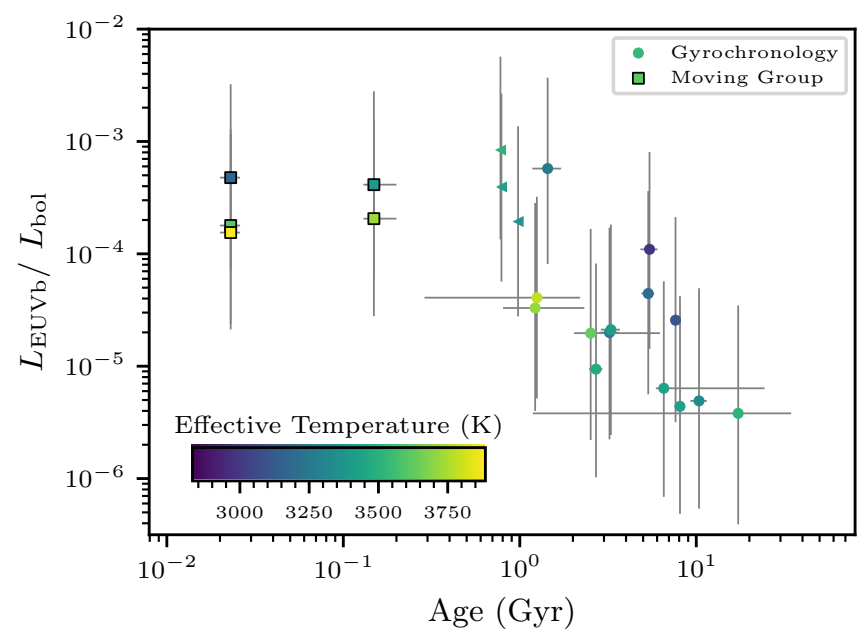

Figure 15. Based on available scaling relations from the FUV (France et al. 2018), we can estimate the EUVb (90-360 ̊) luminosity of low-mass stars, similar to Figure 14. The temporal evolution of EUV emission drops $\sim 2$ orders of magnitude from youth to field ages, see Section 5.

While these empirical relations for age, and difficult to detect portions of the spectrum, like the EUV, provide general estimates for the evolution of activity and high-energy emission, Figure 15 further illustrates the broad uncertainty inherent in using these estimates as inputs to exoplanetary evolution modeling. Refined rotation-age relations in the Mdwarf regime, and expanded samples of stellar EUV detections, from missions like ESCAPE (France et al. 2019), will greatly expand the utility of these methods for exoplanetary applications.

Assuming the Rossby parameterization removes a significant mass dependence, we use the rotation-activity correlation regression fits together with the assumed rotation evolution to calculate the activity evolution of early and mid Mdwarfs. In Figure 16, we plot separate curves for the evolution of Nv emission with stellar age, for a $0.6 M_{\odot} \mathrm{M}$-dwarf, corresponding to the M0-M1 rotational evolution, and a 0.25 $M_{\odot} \mathrm{M}$-dwarf, corresponding to the M2.5-M6 rotational evolution. The top panel of Figure 16, shows the expected median evolution for each mass star with its central $68 \%$ confidence level. The uncertainties are determined at a given age by sampling the uncertainty distributions of the rotation-age relation, the calibration between mass and convective turn over time, and the joint posterior of the rotation-activity fit. The bottom panel of Figure 10 additionally includes the scatter at fixed Rossby number (see Section 4), to illustrate the range of likely emission values accounting for intrinsic scatter in the observed population. More data are required to assess; however, we expect this scatter to include the effects of rotational variability, activity cycles, and possible metallicity variations. While the nominal $\mathrm{Nv}$ emission levels relative to 


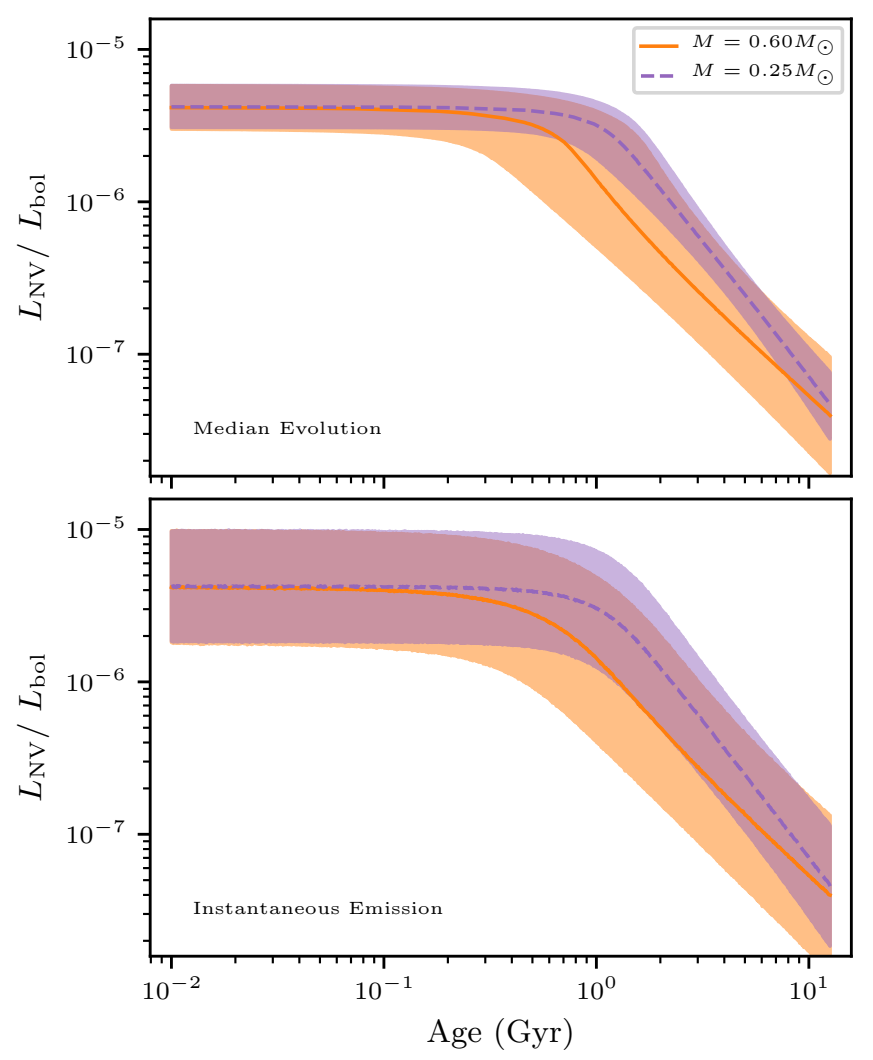

Figure 16. The early and mid M-dwarf FUV temporal evolution implied by our rotation-activity correlation in $\mathrm{Nv}$, and the empirical rotation-age relations, show possible differences across time, with ranges indicating the central $68 \%$ confidence interval about the median. The top panel focuses on just the average evolution, and the bottom panel includes the likely range for emission accounting for the observed scatter across the populations $(\sim 0.33 \mathrm{dex})$.

bolometric are higher for lower mass objects at the same age, there is little difference in the evolution within the uncertainties.

\subsection{Accumulated UV Evolutionary Histories}

With possibly distinct UV luminosity evolution between early and mid M-dwarfs, as shown in Figure 16, we calculate the typical evolution of the UV illumination for exoplanetary systems using the median $\mathrm{Nv}$ rotation-activity relation (Section 4), and the age-rotation evolution from (Engle \& Guinan 2018). The average evolution for individual systems may vary by up to a factor of two. As a measure of the illumination histories, we assess how much quiescent UV energy a given exoplanet accumulates over time as

$$
\mathcal{E}(t)=\int_{0}^{t} F_{\mathrm{UV}}(\tau) d \tau=\int_{0}^{t} \frac{\mathcal{R}(\tau) L_{\mathrm{bol}}(\tau)}{4 \pi d^{2}} d \tau,
$$

where $F$ is the UV flux impinging on the planet at an average distance $d$ from the host, and we integrate from 0 to an age $t$. The UV flux can be rewritten as a combination of the emis- sion ratio evolution, $\mathcal{R}(\tau)=L_{y} / L_{\mathrm{bol}}$, determined from Section 4 , and $L_{\text {bol }}(\tau)$, which accounts for changes in bolometric luminosity with stellar evolution. In evaluating Equation 4, we use evolutionary models (Dotter et al. 2008; Feiden 2016) for the bolometric luminosity, which can evolve substantially at young ages (see Figure 17), and allows a self-consistent metric across several Gyrs. We compared the accumulated UV energies for planets around a $0.25 M_{\odot}$ host and a 0.60 $M_{\odot}$ host with that of a planet around a Sun-like star.

In order to compare with the lower-mass objects, the UV emission history for Sun-like stars required a different treatment from our approach with M-dwarfs. Using a sample of G-dwarfs, Ribas et al. (2005) determined the EUV evolutionary history of Sun-like stars, as a simple power-lay decay with time across 0.1-6.7 Gyr. Since results from France et al. (2018) suggest that the Nv emission and EUV of solar and lower-mass stars are related by a simple multiplicative factor, we consider the Sun-like Nv emission to follow the same power-law defined by Ribas et al. (2005). We thus scaled their result to the Sun's current Nv luminosity from Duvvuri et al. (accepted) using the solar minimum quiescent Solar irradiance spectrum of Woods et al. (2009):

$$
F_{\mathrm{Nv}, \odot}=0.105 \tau^{-1.23}, \mathrm{erg} \mathrm{s}^{-1} \mathrm{~cm}^{-2}, 0.1<\tau<6.7 \mathrm{Gyr}
$$

where $\tau$ is in units of Gyrs. We take the current age of the Sun to be $4.6 \mathrm{Gyr}$, and this flux is evaluated at a distance of 1 AU. Although the EUV bands from Ribas et al. (2005) and France et al. (2018) are different, this conversion allows a representative evolutionary history for the Sun-like Nv emissions, which we can compare with the activity-age evolution determined for M-dwarfs in Section 5.1. Since Equation 5 is only applicable to a particular age interval, to extrapolate to younger ages we consider the UV emissions at $\tau=0.1 \mathrm{Gyr}$ to correspond to a saturated level relative to bolometric that is constant for $\tau<0.1 \mathrm{Gyr}, \log L_{\mathrm{Nv}} / L_{\mathrm{bol}}=-5.75$, set by the model bolometric luminosity at $\tau=0.1 \mathrm{Gyr}$. We then let the bolometric luminosity change according to the non-magnetic Dartmouth stellar models for $\tau<0.1$ Gyr (e.g., Dotter et al. 2008).

We show our results in Figure 18, as the accumulated Nv emission experienced by a planet at a fixed distance of $1 \mathrm{AU}$ from its host, for the 3 planet host cases, $0.25 M_{\odot}, 0.60 M_{\odot}$, and $1.0 M_{\odot}$, starting at $1 \mathrm{Myr}$, the lower limit of the evolutionary models. Around the higher-mass objects the absolute $\mathrm{Nv}$ illumination is typically higher than around the lowermass stars. The shaded bands in Figure 18 propagate the uncertainty in the median evolution (as in Figure 16) through the integral of Equation 4. We do not include the intrinsic scatter because we focus here on the cumulative average history from the rotation-activity analysis. Our treatment, however, excludes uncertainty from unknown systematics with 


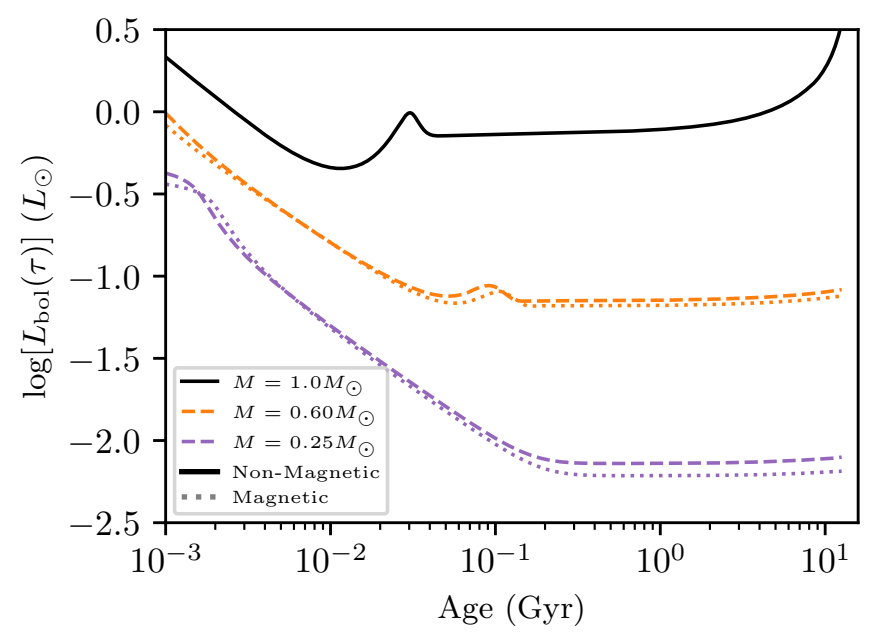

Figure 17. The bolometric luminosity evolution in the stellar models used in this work to calculate the history of low-mass star UV illumination.

the bolometric luminosity evolution, although at least with regards to the role of magnetism, its contribution may be minor (see Figure 17). Ribas et al. (2005) did not report uncertainties for their EUV evolution in Sun-like stars, and we thus do not include it either.

In Figure 19, we also show the ratios $\left(\mathcal{E}_{0.60} / \mathcal{E}_{\odot}, \mathcal{E}_{0.25} / \mathcal{E}_{\odot}\right.$, and $\mathcal{E}_{0.25} / \mathcal{E}_{0.60}$ ) of the accumulated UV energies, propagating the uncertainty in the M-dwarf median rotational evolution. The left-side axes give the result for planets at equal distances around each star, and the right-side axes indicate the same ratio, but for the respective habitable zones. This further assumes planets at that distance have remained there throughout history. The right-axis curves are thus a constant multiple of the left-axis, as in

$$
\left.\frac{\mathcal{E}_{a}}{\mathcal{E}_{b}}\right|_{\mathrm{HZ}}=\frac{\int_{0}^{t} \mathcal{R}_{a} L_{\mathrm{bol}, a} d \tau}{\int_{0}^{t} \mathcal{R}_{b} L_{\mathrm{bol}, b} d \tau} \times \frac{d_{\mathrm{HZ}, b}^{2}}{d_{\mathrm{HZ}, a}^{2}},
$$

where the habitable zone distances are determined by the 5 Gyr bolometric instellations. The right-side axes in Figure 19 thus indicate the relative accumulated UV energies for planets in the respective field age habitable zones of each star.

In Figure 19, we illustrate in the low-mass star regime that at a constant distance exoplanets orbiting higher-mass objects experience a greater absolute accumulation of UV energy. However, within the respective habitable zones around each host the planets orbiting the lower-mass star intercept much more UV energy. For example, relative to the Earth around the Sun, the same planet in the habitable zone around the $0.25 M_{\odot}$ star will accumulate $\sim 20$ times more UV energy by the time it has reached field age, which is $\sim 2$ times more than the same planet in the habitable zone around the $0.60 M_{\odot}$ star. Within the known uncertainty of the median rotational

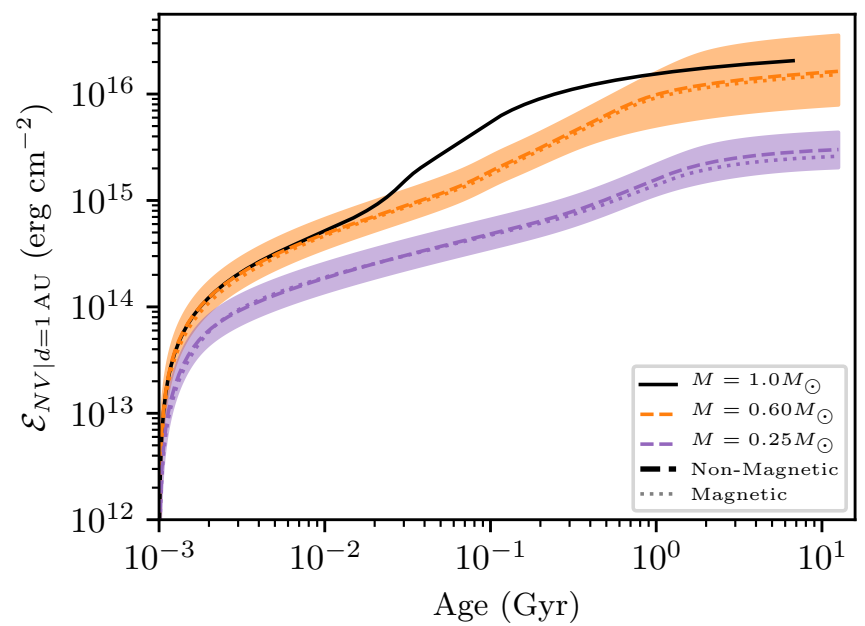

Figure 18. The cumulative $\mathrm{Nv}$ energy experienced by a planetary system at a distance of $1 \mathrm{AU}$ from its host star, according to Equation 4 , for each of 3 host masses, accounting for rotation-activity and bolometric luminosity evolution. For the M-dwarfs we include two lines each to illustrate the evolution with magnetic and nonmagnetic models. The absolute high-energy fluxes at other bands can be scaled from the $\mathrm{Nv}$ emissions. The shaded bands indicate the central $68 \%$ confidence interval for the median evolution of the UV emissions using non-magnetic models, the corresponding magnetic model interval (not shown) is similar.

evolution these figures can vary by factors of a couple. The curves of Figure 19 reflect at early times differential bolometric luminosity evolution modulated by activity-age evolution at late times, with modulations of the evolution dictated by the age at which each star begins their power-law decay of UV activity. The bottom panel of Figure 19 also shows the results using both magnetic (dashed line) and non-magnetic (solid line) models to illustrate that similarity of the luminosity evolution, and the robustness of our results to this potential systematic effect.

These results were derived specifically using Nv emissions; however, the line is broadly representative of the entire FUV band given the strong correlations between emission lines (Section 3.4). Thus, although the absolute energy levels determined from Equation 4, and shown in Figure 18, will vary between choice of UV feature, the ratios of Figure 19 are broadly representative of total FUV emissions. Moreover, to the extent that the $\mathrm{Nv}$ emission is directly proportional to EUV emissions (France et al. 2018), the ratios of Figure 19 translate exactly to the relative exposure of planets to EUV emissions around each stellar host. This is a key result given the importance of EUV fluxes for exoplanetary atmospheric heating and escape.

In Figure 20, we further show the importance of different epochs in the total accumulated UV exposure for exoplanetary systems. Relative to the cumulative quiescent UV 


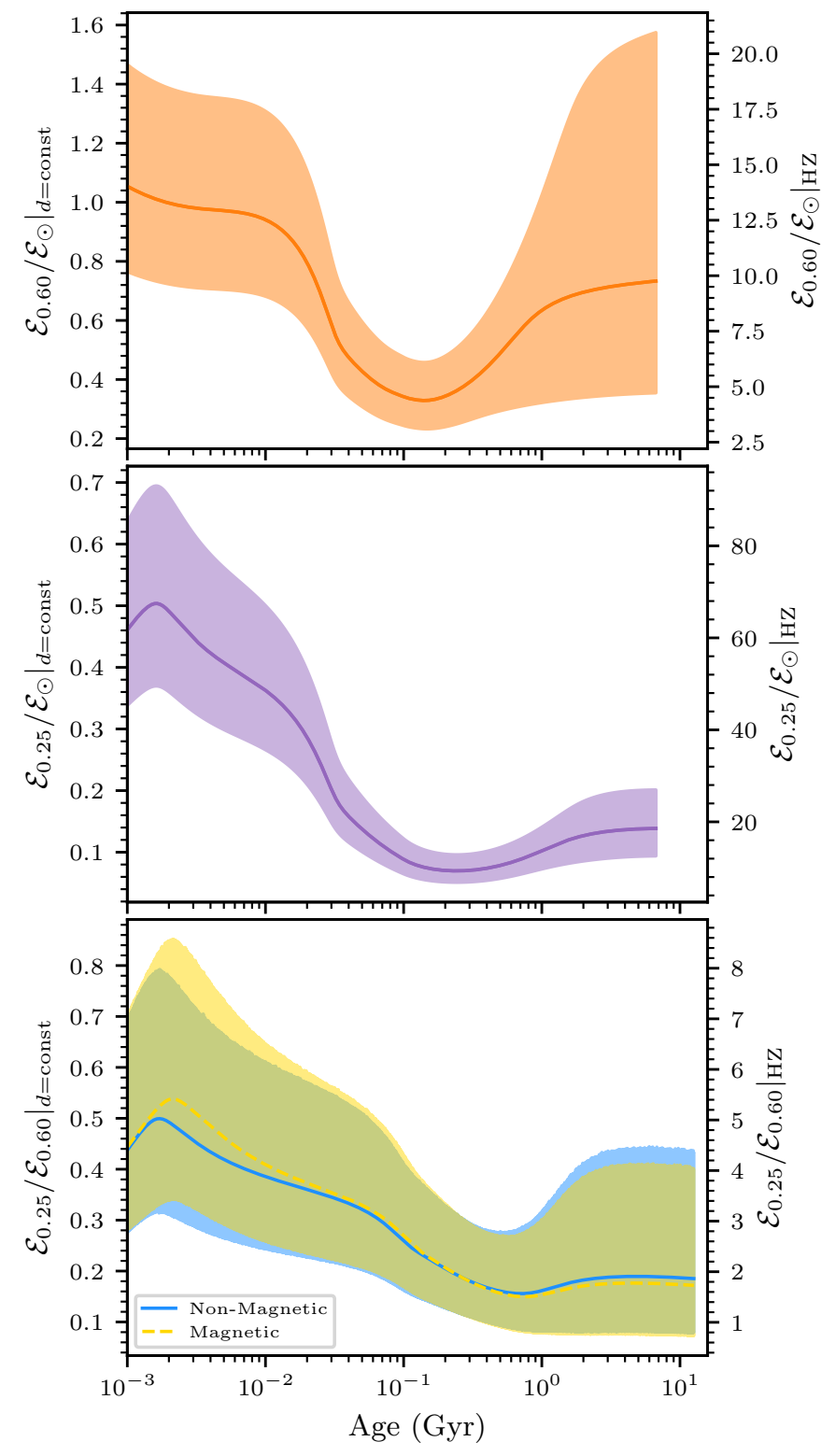

Figure 19. A ratio comparison of the accumulated UV energies over evolutionary history impacting planets orbiting stars of 0.25 , 0.60 and $1.0 M_{\odot}$, following Equation 4 . The left-side axes correspond to planets at equal distances around the respective hosts, and the right-side axes correspond to planet distances at which the $5 \mathrm{Gyr}$ bolometric instellations are equivalent, i.e., comparing the field-age habitable zones around each host. Top - Early-M dwarf relative to Sun-like star, middle - mid-M dwarf relative to Sun-like star, and Bottom - mid-M dwarf relative to early-M dwarf, using both magnetic (dashed) and non-magnetic (solid) models for the stellar luminosity evolution, see Section 5.2. In each case, the more massive hosts deliver more UV energy to planets at the same distance, but within their respective habitable zones, planets orbiting the lowermass star experience greater levels of UV emission. The shaded regions of each panel indicate the central $68 \%$ confidence region accounting for the known uncertainty in the median rotational evolution of FUV emissions.

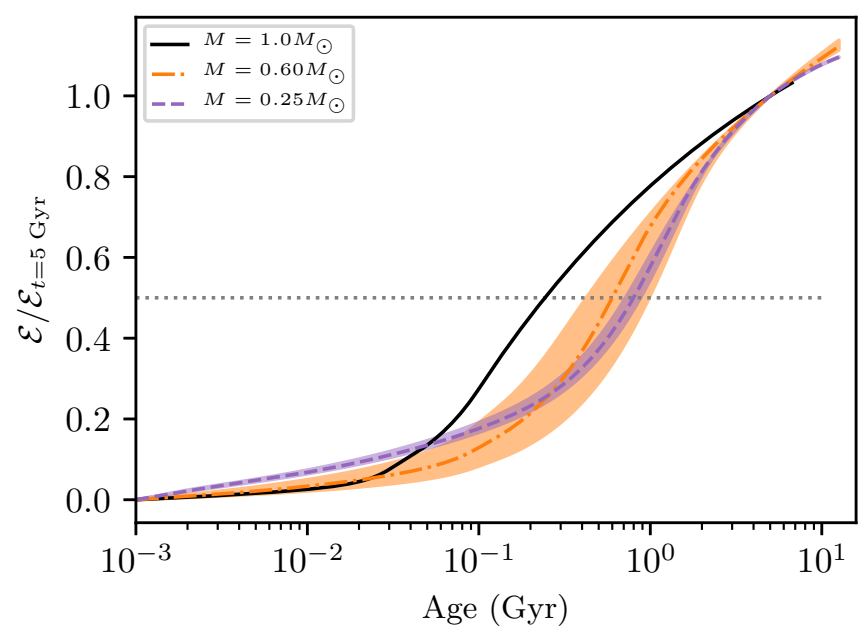

Figure 20. The UV energy accumulation (see Equation 4) as a function of time (as in Figure 18) relative to the total at $t=5 \mathrm{Gyrs}$, using non-magnetic stellar evolutionary models. During the first Gyr of their lifetimes, planets around each low-mass stellar host receive at least $50 \%$ of the total quiescent UV energy exposure that will have accumulated by an age of $5 \mathrm{Gyr}$.

energy experienced at an age of 5 Gyrs, for planets around low-mass hosts, they reach total energetic exposures exceeding $\sim 50 \%$ by ages of 800,600 , and 250 Myr respectively for hosts of mass $0.25,0.60$, and $1.0 M_{\odot}$. These results quantify the importance of the first Gyr of stellar lifetimes in their total energetic input to exoplanetary systems. These early ages clearly need to be taken into account when considering the evolutionary history of planetary systems, and their response to high-energy emissions.

\section{CONCLUSIONS}

The UV data of the FUMES sample presented in this paper combined with the literature data has allowed us to examine how the far ultraviolet spectroscopic emissions of low-mass stars change with angular momentum evolution over time. At fast rotation rates (Rossby number, $R o \lesssim 0.2$ ), corresponding to young stellar ages $(\lesssim 1)$ Gyr, the FUV lines exhibit saturated emissions at levels of $10^{-3.5}-10^{-5.4}$ relative to the stellar bolometric luminosity, depending on the specific line, with Ly $\alpha$ being the strongest feature. As the stars spin-down with age, these emission levels drop by $\sim 2$ orders of magnitude for typical field ages. However, this decline appears to be weakest in $\operatorname{Ly} \alpha$, which will change the spectroscopic balance of energy output (i.e., FUV/NUV ratio) between young and old M-dwarfs. This evolutionary behavior is evident throughout the M-dwarf sample for both early and mid-to-late M-dwarfs. Because these stars show similar FUV luminosities relative to bolometric, the early M-dwarfs are more UV luminous in absolute terms, but with potentially different spin-down behaviors, the cooler stars may emit at the saturation levels for 
a greater duration of their early lifetimes. This evolutionary behavior may have several implications across stellar astrophysics and exoplanetary science.

\subsection{Stellar Atmospheres and Dynamos}

The FUV emission lines directly probe the transition region of the stellar coronal atmosphere. Our spectroscopic data showed how the different features change with rotation, directly implying how the atmospheric structure changes across time, between active and inactive low-mass stars. These data can thus be used to directly assess those changes through stellar chromospheric and coronal models (e.g., Fontenla et al. 2016; Peacock et al. 2019). The comparison of the rotation-activity relationships across $\mathrm{H} \alpha$, the FUV, and $\mathrm{X}$-rays, however, suggests more significant changes in the corona with spin-down relative to the changes in the deeper layers of the atmosphere. These differences must be a direct consequence of how the non-thermal heating processes change with rotation rate. Models of the magnetic heating process itself in M-dwarfs must be able to account for these evolutionary effects, and their significance at different layers of the atmosphere.

This aspect of non-thermal chromospheric/coronal heating is an important consideration in making dynamo inferences, because the observed rotation-activity relationships in different wavebands, often used for this endeavor, are mediated by the magnetic heating, and are not directly defined by the dynamo processes. The multi-wavelength rotation-activity relationships need to be reconciled with more homogeneous methodologies, including the possibly different behaviors in different upper atmospheric layers in order to provide definitive conclusions with respect to dynamo theory. Crucially, while our analysis shows that a Rossby scaling works well for normalizing the rotation-activity relationships in both partly and fully convective M-dwarfs, the empirical scaling may be masking real differences in these populations. This effect is potentially evident in alternative scalings, however, larger samples are required to examine these differences in the UV, to compare with other wavebands (e.g., Magaudda et al. 2020).

\subsection{Exoplanets}

The stellar high-energy spectrum largely defines the prevalent photochemistry and mass-loss history of exoplanetary systems. While these effects have often been investigated in the context of individual nearby planets around low-mass stars, the present day observations of the planetary atmosphere have been shaped by the cumulative history of these stellar emissions. Our spectroscopic FUV data provide its rotational evolution for M-dwarfs directly, which can be transformed using rotation-age gyrochronology relationships. Although the latter remain uncertain for M-dwarfs, their im- provement will greatly improve our assessments of this evolutionary history. Employing literature scaling relations using FUV emission features then enables estimates across the high-energy spectrum, including the EUV (e.g., France et al. 2016, 2018). To understand exoplanetary atmospheres this stellar emission history needs to be taken into account.

Of particular importance is the likely difference between early and mid-to-late M-dwarfs, with regard to how long they persist in exhibiting near saturation level activity. By old field ages, planets in similar orbits around these two different kinds of hosts will have experienced likely different histories in high-energy radiative environments (e.g., Luger \& Barnes 2015). Moreover, changes in the relative significance of FUV or NUV emissions over time will influence the prevalent exoplanetary atmospheric molecules that are observable today. Our results enable a way to account for these effects across different emission features and wavebands when considering new exoplanetary systems.

Using our rotation-activity correlation fits (Section 4), assuming no residual mass dependence, we can predict the most prominent FUV emission features in quiescence from a known rotation period and mass to generally within 0.3 dex of intrinsic scatter. This scatter pertains to the sample population and is likely a consequence of the combined effects of activity cycles, rotation variations in visible active regions, metallicity differences, and/or the stochastic nature of magnetic heating. As an example, we imagine a $0.4 M_{\odot}$ star with $60 \mathrm{~d}$ rotation period, with $3 \%$ uncertainty on the mass and 5\% in the rotation period. Accounting for scatter in the Rossby number calibration and our best fit parameters, our rotation-activity correlations would predict a mean value of $\log \left(L_{\mathrm{CIV}} / L_{\mathrm{bol}}\right)=-6.21 \pm 0.16$. With improved rotationage relations, our data will enable a more comprehensive assessment of the high-energy radiative input to exoplanetary systems across time.

\section{SUMMARY}

In this paper, we have examined the far ultraviolet emission of M-dwarf stars, as probes of the stellar upper atmosphere and non-thermal magnetic heating, their rotational evolution, and possible implications for planetary systems orbiting these kinds of hosts. Additional FUMES papers will discuss the Ly $\alpha$ reconstructions (Youngblood et. al. accepted), and time variability in the UV/optical emissions (Duvvuri et al. in prep). For this work, our primary findings are summarized below.

- We reported emission line-emission line correlations across $\sim 3$ orders of magnitude for the FUV features with respect to Civ emissions, revealing $\sim 0.1-0.2$ dex of intrinsic scatter between FUV features defining the extent to which such features can be used to predict 
one another across the M-dwarf population, see Section 3.4 .

- We provided rotation-activity correlations as a function of Rossby number across 8 UV features, including Ly $\alpha$, with typical power-law slopes of -2 and critical Rossby numbers of 0.2 , see Section 4.

- The decay of $\operatorname{Ly} \alpha$ emission with rotation is likely weaker than it is for other FUV features, implying evolutionary changes in the relative balance of UV spectroscopic emissions, see Section 4.2.

- A possible trend in the rotation-activity correlations as a function of atmospheric layer points to the importance of disentangling magnetic heating effects through the stellar atmosphere when investigating the dynamo dependence on rotation rate, see Section 4.3.

- We demonstrated systematic effects in the resulting fit parameters for rotation-activity correlations (powerlaw slope, critical Rossby number) when utilizing different empirical calibrations for the convective turn over time as a function of mass, see Appendix B.

- Mid-to-late M-dwarfs may exhibit saturation level FUV activity for a longer duration of their early lifetimes relative to early $\mathrm{M}$-dwarfs, with correspondingly distinct histories of high-energy emission impacting exoplanetary systems around these hosts, see Section 5.

- Planets in the habitable zones around mid-to-late Mdwarfs, at field ages, will have accumulated $\sim 2 \times$ more EUV exposure than planets around early M-dwarfs, and $20 \times$ more exposure than planets in the habitable zones around Sun-like stars, see Section 5.2.

- For planets orbiting low-mass stars, the majority of energetic UV exposure accumulated by the age of 5 Gyrs was experienced during the saturated phase of activity evolution, lasting $\sim 1 \mathrm{Gyr}$, see Section 5.2.

\section{ACKNOWLEDGMENTS}

The authors would like to thank Zachary Berta-Thompson, Elisabeth Newton, and Girish Duvvuri for useful discussions and commentary in the preparation of this work. The authors also acknowledge R. O. Parke Loyd and Alejandro Nuñez for providing additional information used in this paper. We further thank the anonymous referee for their comments in the preparation of this publication.

Support for Program numbers HST-GO 14640, 14633 and 15071 were provided by NASA through a grant from the Space Telescope Science Institute, which is operated by the
Association of Universities for Research in Astronomy, Incorporated, under NASA contract NAS5-26555.

A.Y. acknowledges support by an appointment to the NASA Postdoctoral Program at Goddard Space Flight Center, administered by USRA through a contract with NASA.

Based on observations at Cerro Tololo Inter-American Observatory, National Optical Astronomy Observatory (NOAO Prop. ID 2017B-0316 ; PI: J. Pineda), which is operated by the Association of Universities for Research in Astronomy (AURA) under a cooperative agreement with the National Science Foundation.

Based on observations obtained with the Apache Point Observatory 3.5-meter telescope, which is owned and operated by the Astrophysical Research Consortium.

\section{Facility: HST (STIS), Blanco (ARCoIRIS), APO (TSPEC)}

\section{APPENDIX}

\section{A. Concerning the Use of Surface Fluxes}

In Section 3.4, we correlated FUV line flux measurements, illustrating tight relationships between different emission features probing distinct temperatures of the transition region. In the literature these kinds of correlations have been expressed similarly, but instead of luminosity, they have been expressed using the surface flux, normalizing the luminosities by the stellar surface area (e.g., Wood et al. 2005; Youngblood et al. 2017). While this attempts to normalize the emissions accounting for the area of the emitting region to enable comparisons across different kinds of stars, the inclusion of the radii introduces additional uncertainty and increases the error correlations, as the radius uncertainty will dominate the error budget relative to the parallax and flux measurements. ${ }^{15}$ In Figure 21, we illustrate this effect using the same data for Civ and Sirv that comprise the upper right most panel of Figure 5, but transforming the emission measurements to surface flux with the radius determinations from Table 2. The representative $2 \sigma$ error ellipses now are all highly inclined, revealing the strong correlations between the uncertainties in each quantity.

The power-law slope estimated in such line-line correlations using the surface flux should be identical to the luminosity approach used in this work, however, the uncertainties on such estimates do not accurately reflect the nature of the underlying data if they do not account for this correlated error, and are less precisely constrained when accounting for the actual radii uncertainty in the analysis. This additional uncertainty may obscure intrinsic scatter in the analyzed correlations. We therefore recommend luminosity as a currently

\footnotetext{
15 This effect was less significant in the past when parallaxes were not known as precisely (pre-Gaia).
} 


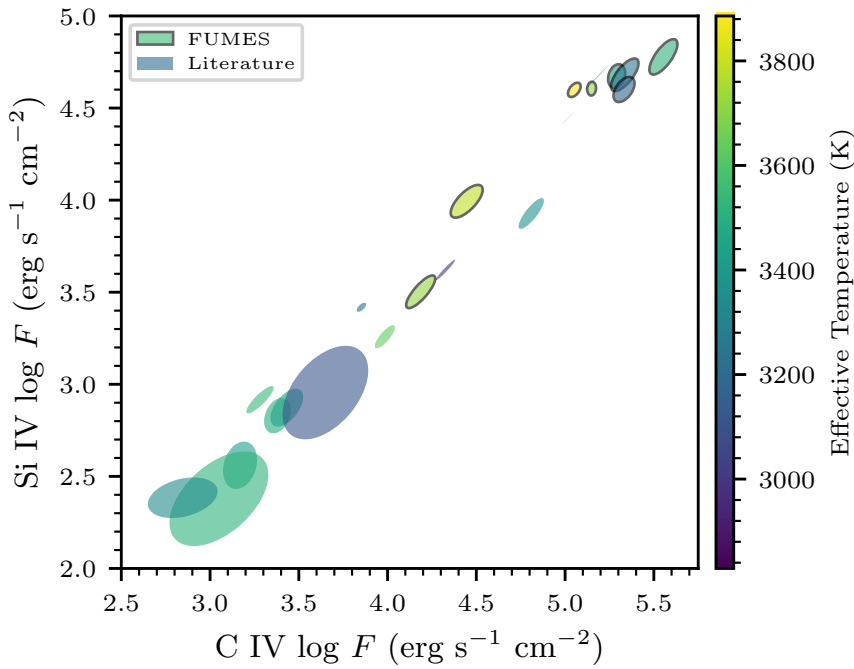

Figure 21. The measured emission strengths of CIV and Sirv as in Figures 5 and 5, but shown as surface flux instead of luminosity utilizing our radius determinations from Table 2. Line-line regression analyzes not accounting for correlated uncertainties, as shown here by slanted ellipses, will yield biased results, see Appendix A.

more robust choice when defining predictive relations between stellar emission features. However, whenever surface fluxes are necessary, a careful accounting of possibly correlations should be included.

\section{B. Systematics with the Convective Turnover Time}

In Section 4, we analyzed the rotation-activity relation of M-dwarfs in FUV emission lines with the FUMES and literature samples. We used the empirical calibration of Wright et al. (2018) to estimate the convective turn over time from the stellar mass in computing the Rossby number, $R o=P / \tau_{c}$, for each star. This empirical calibration is based on minimizing the scatter in the X-ray rotation-activity correlation of low-mass stars. Wright et al. (2011) details the typical procedures used in developing this kind of empirical calibration. Since the $R o$ is generally closely related to the internal dynamo action (although see Reiners et al. 2014), the use of this kind of calibration enables a dynamo comparison amongst stars with convective interiors, from $\mathrm{F}$ to $\mathrm{M}$ stars. However, changes in the kind of magnetic dynamo that generates field in fully convective stars, for example $\alpha^{2}$ instead of $\alpha$ - $\Omega$ (e.g., Browning 2008), suggest that there is no physical reason a single such calibration should work across that full range. Moreover, with relatively deeper convective zones, a single representative value for the time scale of convective motions is likely an increasingly poor approximation with decreasing stellar mass. Accordingly, although the empirical calibration reduces the X-ray rotation-activity scatter, it may not be representative of the dynamo behavior in the fully convective regime.

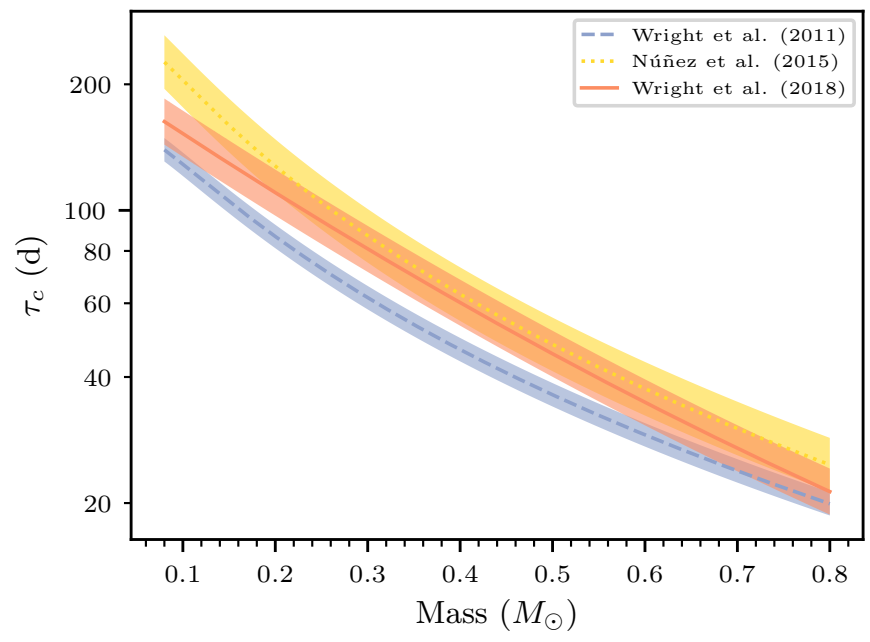

Figure 22. Different calibrations for the convective turn over time as a function of mass yield systematic differences in rotationactivity analyses when used to compute a characteristic Rossby number, Ro, see Appendix B.

Nevertheless, using these relations provides a means to compare to literature results and test how the $\mathrm{X}$-ray calibrated relation applies to the activity at other wavelengths, as we have done in Section 4. As detailed in this Appendix, we further investigated how those results are impacted by the choice of empirical calibration for the convective turnover time as a function of stellar mass. In Figure 22, we plot three such literature calibrations from Wright et al. (2011), Núñez et al. (2015), and Wright et al. (2018), including the scatter about those relationships, reported in those works, or obtained via private communication (0.064 dex, Nuñez, A.). The Núñez et al. (2015) calibration uses the same data as Wright et al. (2011), but assumes the canonical value for the best fit slope $(-2)$ instead of the best fit value from Wright et al. (2011), and the Wright et al. (2018) calibration, which sits in between the other two, updates the Wright et al. (2011) result with more fully convective stars and a slightly different functional form.

We re-fit the rotation-activity data presented in Section 4, using the same methods, but using the two additional literature calibrations to determine their impact on the best fit parameters. These results are shown in Table 9 for the critical Rossby number, $R o_{c}$, and in Table 10 for the slope of the unsaturated regime, $\eta$. The effect of the different calibrations is most readily illustrated by comparing the results using Wright et al. (2011) vs. Núñez et al. (2015), as they have a greater separation in $\tau_{c}-M$ space (see Figure 22). The Núnez et al. (2015) calibration gives higher convective turnover times at a given mass than Wright et al. (2011). Consequently, the best fit $R o_{c}$ is systematically smaller using Núñez et al. (2015) than it is when using the Wright et al. (2011) calibration - higher $\tau_{c}$ corresponds to smaller 
Table 9. Critical Rossby Systematics: $R o_{c} a$

\begin{tabular}{lccc}
\hline \hline Line & Wright et al. (2011) & Núñez et al. (2015) & Wright et al. (2018) \\
\hline Ly $\alpha$ & $0.25 \pm_{0.12}^{0.20}$ & $0.19 \pm_{0.10}^{0.14}$ & $\mathbf{0 . 2 1} \pm_{\mathbf{0 . 1 1}}^{\mathbf{0 . 1 6}}$ \\
MgII & $0.23 \pm_{0.09}^{0.14}$ & $0.19 \pm_{0.08}^{0.11}$ & $\mathbf{0 . 2 0} \pm_{\mathbf{0 . 0 8}}^{\mathbf{1 . 1 1}}$ \\
CII & $0.28 \pm_{0.14}^{0.15}$ & $0.20 \pm_{0.09}^{0.10}$ & $\mathbf{0 . 2 4} \pm_{\mathbf{0 . 1 1}}^{\mathbf{0 . 1 2}}$ \\
SiIII & $0.23 \pm 0.08$ & $0.18 \pm 0.06$ & $\mathbf{0 . 2 0} \pm \mathbf{0 . 0 7}$ \\
HeII & $0.22 \pm 0.06$ & $0.16 \pm_{0.04}^{0.05}$ & $\mathbf{0 . 1 9} \pm \mathbf{0 . 0 5}$ \\
SiIV & $0.28 \pm 0.08$ & $0.22 \pm 0.06$ & $\mathbf{0 . 2 4} \pm \mathbf{0 . 0 7}$ \\
CIV & $0.22 \pm 0.08$ & $0.17 \pm 0.06$ & $\mathbf{0 . 1 8} \pm_{\mathbf{0 . 0 6}}^{\mathbf{0 . 0 7}}$ \\
Nv & $0.22 \pm 0.09$ & $0.18 \pm 0.06$ & $\mathbf{0 . 1 9} \pm \mathbf{0 . 0 7}$ \\
\hline
\end{tabular}

${ }^{a}$ Reported parameters correspond to the median of the marginalized posterior distribution with uncertainties indicating the central $68 \%$ confidence interval. The reference for each column indicates the source used for the calibration of the mass dependent convective turn over time.

Table 10. Unsaturated Slope Systematics: $\eta^{a}$

\begin{tabular}{lccc}
\hline \hline Line & Wright et al. $(2011)$ & Núñez et al. $(2015)$ & Wright et al. (2018) \\
\hline Ly $\alpha$ & $-1.21 \pm_{0.54}^{0.39}$ & $-1.25 \pm_{0.56}^{0.42}$ & $-\mathbf{1 . 2 6} \pm_{\mathbf{0 . 5 8}}^{\mathbf{0 . 4 1}}$ \\
MgII & $-1.72 \pm_{0.51}^{0.37}$ & $-1.86 \pm_{0.58}^{0.44}$ & $-\mathbf{1 . 8 6} \pm_{\mathbf{0 . 5 5}}^{\mathbf{0 . 4 1}}$ \\
CII & $-2.25 \pm_{0.72}^{0.62}$ & $-2.25 \pm_{0.70}^{0.57}$ & $-\mathbf{2 . 3 8} \pm_{\mathbf{0 . 7 7}}^{\mathbf{0 . 6 4}}$ \\
SiIII & $-1.94 \pm_{0.41}^{0.39}$ & $-2.10 \pm_{0.42}^{0.44}$ & $-\mathbf{2 . 0 8} \pm_{\mathbf{0 . 4 6}}^{\mathbf{0 . 4 1}}$ \\
HeII & $-2.11 \pm_{0.29}^{0.27}$ & $-2.15 \pm_{0.32}^{0.30}$ & $\mathbf{- 2 . 1 9} \pm_{\mathbf{0 . 3 3}}^{\mathbf{0 . 3 0}}$ \\
SiIV & $-2.21 \pm_{0.41}^{0.40}$ & $-2.31 \pm_{0.42}^{0.39}$ & $-\mathbf{2 . 3 2} \pm_{\mathbf{0 . 4 4}}^{\mathbf{0 . 4 2}}$ \\
CIV & $-1.92 \pm_{0.39}^{0.37}$ & $-2.04 \pm_{0.39}^{0.37}$ & $\mathbf{- 2 . 0 2} \pm_{\mathbf{0 . 4 1}}^{\mathbf{0 . 3 9}}$ \\
Nv & $-1.76 \pm_{0.38}^{0.37}$ & $-1.88 \pm_{0.37}^{0.36}$ & $\mathbf{- 1 . 8 4} \pm_{\mathbf{0 . 4 0}}^{\mathbf{0 . 3 7}}$ \\
\hline
\end{tabular}

$a_{\text {Reported parameters correspond to the median of the marginalized poste- }}$ rior distribution with uncertainties indicating the central $68 \%$ confidence interval. The reference for each column indicates the source used for the calibration of the mass dependent convective turn over time.

Ro. Between the two calibrations as applied to our data, this yielded a systematic offset of $\sim 0.04-0.05$ in $R o_{c}$, see Table 9 .

This effect on the best fit critical Rossby number is relatively intuitive given the direct impact on the convective turnover time between calibration choices. However, we also observe a systematic difference in the best fit slope from the rotation-activity analysis when changing between empir- ical calibrations. With our data, larger assumed convective time scales (smaller $R o$ ) yielded systematically steeper slopes (more negative) for the unsaturated regime, see Table 10. Although small, this effect is generally evident across the eight different lines we analyzed. To illustrate this systematic effect, we show representative ellipses for the joint posterior distributions of $R o_{c}-\eta$ across 4 FUV lines in Figure 23. The error ellipses for each individual line shift to the left (smaller $R o_{c}$ ), and down (steeper $\eta$ ) when changing calibrations from Wright et al. (2011) to Núñez et al. (2015). Because these fit parameters are correlated, it is perhaps unsurprising that systematic effects would appear in both $R o_{c}$ and $\eta$, however, the systematic shift is not in the same direction, as the critical Rossby number and slope posteriors are anti-correlated, not correlated.

We attribute this systematic effect to the non-linearity of the calibrations as applied to individual samples. If the choice of empirical calibration scaled the assumed Rossby numbers of all of the stars in the same way, we could expect the best fit slope of the unsaturated regime to remain constant. A comparison of the calibrations for $\tau_{c}(M)$ (see Figure 22), shows that this is generally not the case. Thus, depending on the sample of stars, some objects shift in $R o$ space more than others. A large number of fully convective stars in the sample would likely increase the magnitude of this systematic effect on the best-fit slopes between the calibrations of Wright et al. (2011) and Núñez et al. (2015), as that is where those functions largely diverge. It is therefore difficult to estimate the extent of this systematic effect on the slopes without doing the entirety of the analysis with multiple calibrations for $\tau_{c}$ for each sample of stars. This makes comparisons somewhat more difficult across the literature as methods have been updated and evolved over time, with different stellar samples. Future comparisons across wavebands and samples will greatly benefit from homogeneous analysis methodologies. The framework presented in this paper for the rotation-activity work (Section 4), accounts for known uncertainties across all available data, possible correlations, and includes a measure of the intrinsic scatter within the regression fit. The presence of these systematics effects, especially when using a quantity as uncertain as the convective turnover time scale, also supports the argument in favor of finding simpler descriptions that capture the relevant physics for characterizing the dependence of activity on stellar physical and rotational properties, as discussed in Reiners et al. (2014). We tested some of those methods in Section 4.4.

\section{REFERENCES}

Alonso-Floriano, F. J., Morales, J. C., Caballero, J. A., et al. 2015,

A\&A, 577, A128, doi: 10.1051/0004-6361/201525803
Angus, R., Aigrain, S., Foreman-Mackey, D., \& McQuillan, A.

2015, MNRAS, 450, 1787, doi: 10.1093/mnras/stv423 


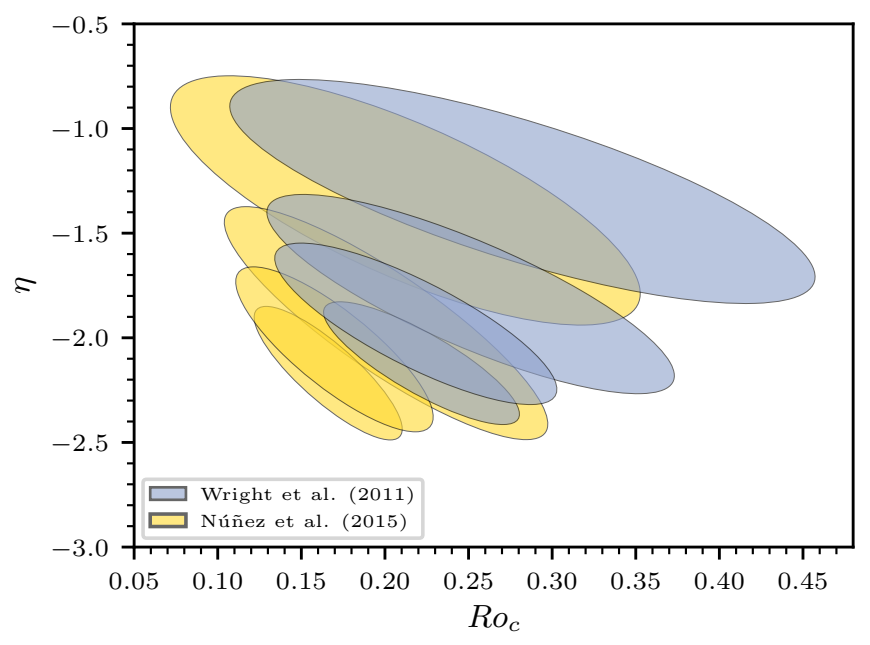

Figure 23. Changing the assumed calibration for convective turnover time as a function of stellar mass when fitting the canonical rotation-activity relationships systematically affects the best fit parameters for the unsaturated slope, $\eta$, and critical Rossby number, $R o_{c}$. The representative error ellipses shown here for four of the FUV line rotation-activity fits collectively shift, yellow (Núñez et al. 2015) relative to blue (Wright et al. 2011), when using distinct calibrations, see Appendix B.

Astudillo-Defru, N., Delfosse, X., Bonfils, X., et al. 2017, A\&A, 600, A13, doi: 10.1051/0004-6361/201527078

Ayres, T. R., Brown, A., Harper, G. M., et al. 2003, ApJ, 583, 963, doi: 10.1086/345409

Barnes, S. A. 2003, ApJ, 586, 464, doi: 10.1086/367639

—. 2010, ApJ, 722, 222, doi: 10.1088/0004-637X/722/1/222

Bell, C. P. M., Mamajek, E. E., \& Naylor, T. 2015, MNRAS, 454, 593, doi: 10.1093/mnras/stv1981

Bourrier, V., Ehrenreich, D., Allart, R., et al. 2017, A\&A, 602, A106, doi: 10.1051/0004-6361/201730542

Bourrier, V., Lovis, C., Beust, H., et al. 2018, Nature, 553, 477, doi: 10.1038/nature24677

Boyajian, T. S., von Braun, K., van Belle, G., et al. 2012, ApJ, 757, 112, doi: 10.1088/0004-637X/757/2/112

Browning, M. K. 2008, ApJ, 676, 1262, doi: 10.1086/527432

Browning, M. K., Miesch, M. S., Brun, A. S., \& Toomre, J. 2006, ApJL, 648, L157, doi: 10.1086/507869

Charbonneau, P., \& MacGregor, K. B. 1997, ApJ, 486, 502, doi: 10.1086/304485

Cushing, M. C., Vacca, W. D., \& Rayner, J. T. 2004, PASP, 116, 362, doi: 10.1086/382907

Donati, J.-F., Morin, J., Petit, P., et al. 2008, MNRAS, 390, 545, doi: 10.1111/j.1365-2966.2008.13799.x

Dotter, A., Chaboyer, B., Jevremović, D., et al. 2008, The Astrophysical Journal Supplement Series, 178, 89, doi: $10.1086 / 589654$
Dressing, C. D., \& Charbonneau, D. 2015, ApJ, 807, 45, doi: 10.1088/0004-637X/807/1/45

Engle, S. G., \& Guinan, E. F. 2018, Research Notes of the American Astronomical Society, 2, 34, doi: 10.3847/2515-5172/aab1f8

Feiden, G. A. 2016, Astronomy and Astrophysics, 593, A99, doi: 10.1051/0004-6361/201527613

Feiden, G. A., \& Chaboyer, B. 2013, The Astrophysical Journal, 779, 183, doi: 10.1088/0004-637X/779/2/183

—. 2014, The Astrophysical Journal, 789, 53, doi: 10.1088/0004-637X/789/1/53

Fontenla, J. M., Linsky, J. L., Witbrod, J., et al. 2016, ApJ, 830, 154, doi: 10.3847/0004-637X/830/2/154

France, K., Arulanantham, N., Fossati, L., et al. 2018, ApJS, 239, 16, doi: 10.3847/1538-4365/aae1a3

France, K., Froning, C. S., Linsky, J. L., et al. 2013, ApJ, 763, 149, doi: 10.1088/0004-637X/763/2/149

France, K., Parke Loyd, R. O., Youngblood, A., et al. 2016, ApJ, 820, 89, doi: 10.3847/0004-637X/820/2/89

France, K., Fleming, B. T., Drake, J. J., et al. 2019, in Society of Photo-Optical Instrumentation Engineers (SPIE) Conference Series, Vol. 11118, Proc. SPIE, 1111808, doi: $10.1117 / 12.2526859$

Gallet, F., \& Bouvier, J. 2015, A\&A, 577, A98, doi: 10.1051/0004-6361/201525660

Gao, P., Hu, R., Robinson, T. D., Li, C., \& Yung, Y. L. 2015, ApJ, 806, 249, doi: 10.1088/0004-637X/806/2/249

Garraffo, C., Drake, J. J., \& Cohen, O. 2015, ApJ, 807, L6, doi: 10.1088/2041-8205/807/1/L6

Garraffo, C., Drake, J. J., Dotter, A., et al. 2018, ApJ, 862, 90, doi: 10.3847/1538-4357/aace5d

Guinan, E. F., Engle, S. G., \& Durbin, A. 2016, ApJ, 821, 81, doi: $10.3847 / 0004-637 X / 821 / 2 / 81$

Hall, J. C. 2008, Living Reviews in Solar Physics, 5, 2 , doi: 10.12942/lrsp-2008-2

Harman, C. E., Schwieterman, E. W., Schottelkotte, J. C., \& Kasting, J. F. 2015, ApJ, 812, 137, doi: 10.1088/0004-637X/812/2/137

Hartman, J. D., Bakos, G. Á., Noyes, R. W., et al. 2011, AJ, 141, 166, doi: 10.1088/0004-6256/141/5/166

Hawley, S. L., Gizis, J. E., \& Reid, I. N. 1996, AJ, 112, 2799 , doi: $10.1086 / 118222$

Hawley, S. L., \& Pettersen, B. R. 1991, ApJ, 378, 725, doi: 10.1086/170474

Hawley, S. L., Walkowicz, L. M., Allred, J. C., \& Valenti, J. A. 2007, PASP, 119, 67, doi: 10.1086/510561

Hawley, S. L., Allred, J. C., Johns-Krull, C. M., et al. 2003, ApJ, 597, 535, doi: 10.1086/378351

Houdebine, E. R., Mullan, D. J., Bercu, B., Paletou, F., \& Gebran, M. 2017, ApJ, 837, 96, doi: 10.3847/1538-4357/aa5cad 
Kane, S. R., von Braun, K., Henry, G. W., et al. 2017, ApJ, 835, 200, doi: 10.3847/1538-4357/835/2/200

Kelly, B. C. 2007, ApJ, 665, 1489, doi: 10.1086/519947

Kirkpatrick, J. D. 2005, ARA\&A, 43, 195, doi: 10.1146/annurev.astro.42.053102.134017

Kirkpatrick, J. D., Henry, T. J., \& McCarthy, Donald W., J. 1991, ApJS, 77, 417, doi: 10.1086/191611

Kirkpatrick, J. D., Henry, T. J., \& Simons, D. A. 1995, AJ, 109, 797, doi: 10.1086/117323

Kirkpatrick, J. D., Reid, I. N., Liebert, J., et al. 1999, ApJ, 519, 802, doi: 10.1086/307414

Klimchuk, J. A. 2006, Solar Physics, 234, 41, doi: 10.1007/s11207-006-0055-z

Küker, M., Rüdiger, G., Olah, K., \& Strassmeier, K. G. 2019, A\&A, 622, A40, doi: 10.1051/0004-6361/201833173

Linsky, J. L. 1980, ARA\&A, 18, 439, doi: 10.1146/annurev.aa.18.090180.002255

-. 2017, ARA\&A, 55, 159, doi: 10.1146/annurev-astro-091916-055327

Linsky, J. L., Worden, S. P., McClintock, W., \& Robertson, R. M. 1979, ApJS, 41, 47, doi: 10.1086/190607

Loyd, R. O. P., \& France, K. 2014, The Astrophysical Journal Supplement Series, 211, 9, doi: 10.1088/0067-0049/211/1/9

Loyd, R. O. P., France, K., Youngblood, A., et al. 2016, ApJ, 824, 102, doi: 10.3847/0004-637X/824/2/102

—. 2018, ApJ, 867, 71, doi: 10.3847/1538-4357/aae2bd

Luger, R., \& Barnes, R. 2015, Astrobiology, 15, 119, doi: 10.1089/ast.2014.1231

Magaudda, E., Stelzer, B., Covey, K. R., et al. 2020, arXiv e-prints, arXiv:2004.02904. https://arxiv.org/abs/2004.02904

Mallonn, M., Herrero, E., Juvan, I. G., et al. 2018, A\&A, 614, A35, doi: 10.1051/0004-6361/201732300

Mann, A. W., Feiden, G. A., Gaidos, E., Boyajian, T., \& von Braun, K. 2015, ApJ, 804, 64, doi: 10.1088/0004-637X/804/1/64

Mann, A. W., Dupuy, T., Kraus, A. L., et al. 2019, ApJ, 871, 63, doi: 10.3847/1538-4357/aaf3bc

Meadows, V. S., Reinhard, C. T., Arney, G. N., et al. 2018, Astrobiology, 18, 630, doi: 10.1089/ast.2017.1727

Meibom, S., Barnes, S. A., Platais, I., et al. 2015, Nature, 517, 589, doi: $10.1038 /$ nature 14118

Messina, S., Desidera, S., Turatto, M., Lanzafame, A. C., \& Guinan, E. F. 2010, A\&A, 520, A15, doi: 10.1051/0004-6361/200913644

Montesinos, B., Thomas, J. H., Ventura, P., \& Mazzitelli, I. 2001, MNRAS, 326, 877, doi: 10.1046/j.1365-8711.2001.04476.x

Morin, J., Donati, J. F., Petit, P., et al. 2008, MNRAS, 390, 567, doi: 10.1111/j.1365-2966.2008.13809.x

Morley, C. V., Kreidberg, L., Rustamkulov, Z., Robinson, T., \& Fortney, J. J. 2017, ApJ, 850, 121, doi: 10.3847/1538-4357/aa927b
Narain, U., \& Ulmschneider, P. 1996, SSRv, 75, 453, doi: 10.1007/BF00833341

Newton, E. R., Charbonneau, D., Irwin, J., et al. 2014, AJ, 147, 20, doi: 10.1088/0004-6256/147/1/20

Newton, E. R., Charbonneau, D., Irwin, J., \& Mann, A. W. 2015, ApJ, 800, 85, doi: 10.1088/0004-637X/800/2/85

Newton, E. R., Irwin, J., Charbonneau, D., et al. 2017, ApJ, 834, 85, doi: 10.3847/1538-4357/834/1/85

—. 2016, ApJ, 821, 93, doi: 10.3847/0004-637X/821/2/93

Newton, E. R., Mondrik, N., Irwin, J., Winters, J. G., \&

Charbonneau, D. 2018, AJ, 156, 217,

doi: 10.3847/1538-3881/aad73b

Noyes, R. W., Hartmann, L. W., Baliunas, S. L., Duncan, D. K., \& Vaughan, A. H. 1984, ApJ, 279, 763, doi: 10.1086/161945

Núñez, A., Agüeros, M. A., Covey, K. R., et al. 2015, ApJ, 809, 161, doi: 10.1088/0004-637X/809/2/161

Owen, J. E., \& Jackson, A. P. 2012, MNRAS, 425, 2931, doi: 10.1111/j.1365-2966.2012.21481.x

Parker, E. N. 1993, ApJ, 408, 707, doi: 10.1086/172631

Peacock, S., Barman, T., Shkolnik, E. L., et al. 2019, ApJ, 886, 77, doi: 10.3847/1538-4357/ab4f6f

Pizzolato, N., Maggio, A., Micela, G., Sciortino, S., \& Ventura, P. 2003, A\&A, 397, 147, doi: 10.1051/0004-6361:20021560

Ranjan, S., Wordsworth, R., \& Sasselov, D. D. 2017, ApJ, 843, 110, doi: 10.3847/1538-4357/aa773e

Rayner, J. T., Cushing, M. C., \& Vacca, W. D. 2009, ApJS, 185, 289, doi: 10.1088/0067-0049/185/2/289

Redfield, S., \& Linsky, J. L. 2002, ApJS, 139, 439, doi: $10.1086 / 338650$

—. 2004, ApJ, 613, 1004, doi: 10.1086/423311

Reid, I. N., Hawley, S. L., \& Gizis, J. E. 1995, AJ, 110, 1838, doi: 10.1086/117655

Reiners, A., \& Mohanty, S. 2012, ApJ, 746, 43, doi: 10.1088/0004-637X/746/1/43

Reiners, A., Schüssler, M., \& Passegger, V. M. 2014, ApJ, 794, 144, doi: 10.1088/0004-637X/794/2/144

Ribas, I., Guinan, E. F., Güdel, M., \& Audard, M. 2005, ApJ, 622, 680, doi: 10.1086/427977

Rojas-Ayala, B., Covey, K. R., Muirhead, P. S., \& Lloyd, J. P. 2012, ApJ, 748, 93, doi: 10.1088/0004-637X/748/2/93

Rutten, R. G. M., Schrijver, C. J., Zwaan, C., Duncan, D. K., \& Mewe, R. 1989, A\&A, 219, 239

Scalo, J., Kaltenegger, L., Segura, A. G., et al. 2007, Astrobiology, 7, 85, doi: 10.1089/ast.2006.0125

Schneider, A. C., \& Shkolnik, E. L. 2018, AJ, 155, 122, doi: 10.3847/1538-3881/aaaa24

Shkolnik, E., Liu, M. C., \& Reid, I. N. 2009, ApJ, 699, 649, doi: 10.1088/0004-637X/699/1/649

Shkolnik, E. L., \& Barman, T. S. 2014, AJ, 148, 64, doi: 10.1088/0004-6256/148/4/64 
Shulyak, D., Reiners, A., Engeln, A., et al. 2017, Nature Astronomy, 1, 0184, doi: 10.1038/s41550-017-0184

Skumanich, A. 1972, ApJ, 171, 565, doi: 10.1086/151310

Stelzer, B., Marino, A., Micela, G., López-Santiago, J., \& Liefke, C. 2013, MNRAS, 431, 2063, doi: 10.1093/mnras/stt225

Suárez Mascareño, A., Rebolo, R., \& González Hernández, J. I. 2016, A\&A, 595, A12, doi: 10.1051/0004-6361/201628586

Suárez Mascareño, A., Rebolo, R., González Hernández, J. I., \& Esposito, M. 2015, MNRAS, 452, 2745, doi: 10.1093/mnras/stv1441

Terrien, R. C., Mahadevan, S., Deshpande, R., \& Bender, C. F. 2015, ApJS, 220, 16, doi: 10.1088/0067-0049/220/1/16

Tian, F., \& Ida, S. 2015, Nature Geoscience, 8, 177, doi: $10.1038 /$ ngeo 2372

Torres, C. A. O., Quast, G. R., da Silva, L., et al. 2006, A\&A, 460, 695, doi: 10.1051/0004-6361:20065602

Vacca, W. D., Cushing, M. C., \& Rayner, J. T. 2003, PASP, 115, 389, doi: 10.1086/346193

van Saders, J. L., Ceillier, T., Metcalfe, T. S., et al. 2016, Nature, 529, 181, doi: 10.1038/nature16168

Vanderburg, A., Rowden, P., Bryson, S., et al. 2020, ApJL, 893, L27, doi: 10.3847/2041-8213/ab84e5

Vidotto, A. A., Gregory, S. G., Jardine, M., et al. 2014, MNRAS, 441, 2361, doi: 10.1093/mnras/stu728

von Braun, K., Boyajian, T. S., Kane, S. R., et al. 2011, ApJL, 729, L26, doi: 10.1088/2041-8205/729/2/L26 von Braun, K., Boyajian, T. S., van Belle, G. T., et al. 2014, MNRAS, 438, 2413, doi: 10.1093/mnras/stt2360

Wahhaj, Z., Liu, M. C., Biller, B. A., et al. 2011, ApJ, 729, 139, doi: 10.1088/0004-637X/729/2/139

Wilson, J. C., Henderson, C. P., Herter, T. L., et al. 2004, in Proc. SPIE, Vol. 5492, Ground-based Instrumentation for Astronomy, ed. A. F. M. Moorwood \& M. Iye, 1295-1305, doi: $10.1117 / 12.550925$

Wood, B. E., Redfield, S., Linsky, J. L., Müller, H.-R., \& Zank, G. P. 2005, The Astrophysical Journal Supplement Series, 159, 118, doi: 10.1086/430523

Woods, T. N., Chamberlin, P. C., Harder, J. W., et al. 2009, Geophys. Res. Lett., 36, L01101, doi: 10.1029/2008GL036373

Wright, N. J., \& Drake, J. J. 2016, Nature, 535, 526, doi: 10.1038/nature18638

Wright, N. J., Drake, J. J., Mamajek, E. E., \& Henry, G. W. 2011, ApJ, 743, 48, doi: 10.1088/0004-637X/743/1/48

Wright, N. J., Newton, E. R., Williams, P. K. G., Drake, J. J., \& Yadav, R. K. 2018, MNRAS, 479, 2351, doi: 10.1093/mnras/sty1670

Youngblood, A., France, K., Loyd, R. O. P., et al. 2016, ApJ, 824, 101, doi: 10.3847/0004-637X/824/2/101

—. 2017, ApJ, 843, 31, doi: 10.3847/1538-4357/aa76dd

Zuckerman, B., \& Song, I. 2004, ARA\&A, 42, 685, doi: 10.1146/annurev.astro.42.053102.134111 